Document downloaded from:

http://hdl.handle.net/10251/154796

This paper must be cited as:

Bravo, R.; Ortiz, P.; Pérez-Aparicio, JL. (2018). Analytical and discrete solutions for the incipient motion of ellipsoidal sediment particles. Journal of Hydraulic Research. 56(1):2943. https://doi.org/10.1080/00221686.2017.1289263

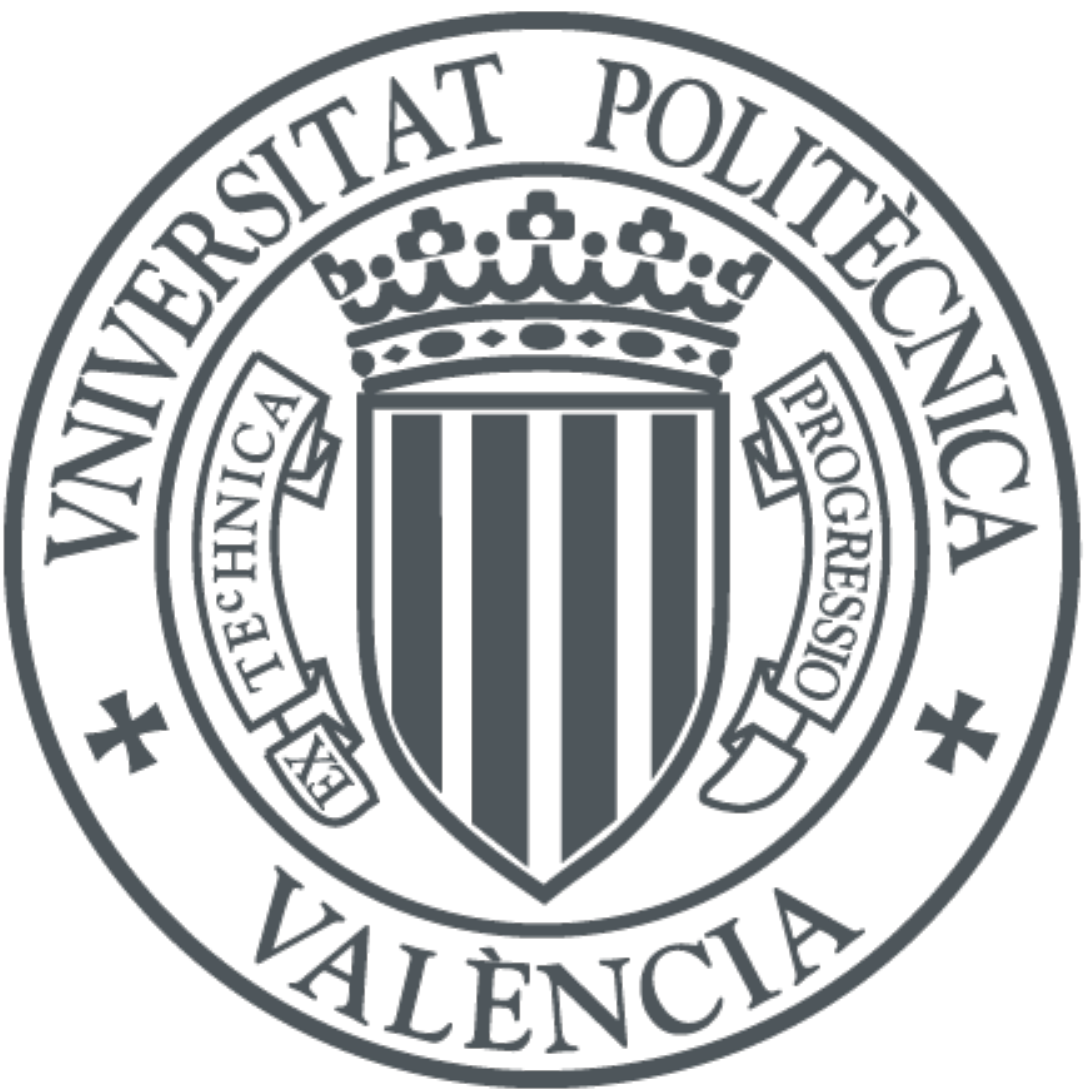

The final publication is available at

https://doi.org/10.1080/00221686.2017.1289263

Copyright Taylor \& Francis

Additional Information 
Research paper

\title{
Analytical and discrete solutions for the incipient motion of ellipsoidal sediment particles
}

RAFAEL BRAVO, Lecturer, University of Granada, Esc. Ing. Caminos. Campus Fuentenueva, 18071

Granada, Spain

Email: rbravo@ugr.es

PABLO ORTIZ, Associate Professor, University of Granada, Esc. Ing. Caminos. Campus Fuentenueva, 18071 Granada, Spain

Email: portiz@ugr.es

JOSÉ LUIS PÉREZ-APARICIO, Associate Professor, Dep. of Continuum Mechanics and theory of Structures, Universitat Politècnica de València 46022 Valencia, Spain

Email: jopeap@upvnet.upv.es

\begin{abstract}
This work introduces analytical and numerical approaches to compute the incipient motion of ellipsoidal sediment particles. Initiation of motion of spherical particles is dominated by rolling mode. However, solutions for initiation of motion for non-spherical grains have to incorporate rolling, sliding, and mixed modes. The proposed approaches include wide variety of shapes and inclinations, that represent realistic configuration of sediment bed layers. The numerical procedure is based on the discrete element method, simulating the micro-mechanics of the sediment as an aggregate of rigid ellipsoids interacting by contact. Numerical discrete solution covers a range of incipient movements that can not be covered by the analytical approach. Hence, some trapped modes observed in analytical calculations are complemented by the numerical computation of threshold stresses. Main results are organised as novel extended Shields diagrams for non-spherical grains, where non-dimensional critical shear stress is represented in terms of friction Reynolds number.
\end{abstract}

Keywords: Bedload; Computational methods in hydro-environment research and fluid dynamics; Discrete element method; Ellipsoidal particles; Fluid-particle interactions

\section{Introduction}

Initiation of motion of a grain by the action of flow on a sedimentary bed is a subject of long research, treated since Shields (1936), and Yalin and Karahan (1979), to analytical works of Wiberg and Smith (1985), James (1990), and Dey (1999), or to recent numerical simulations by Bravo, Ortiz, and Pérez-Aparicio (2014). Main purpose is to establish the critical shear stress to give rise to particle motion. Analytical approaches determine the dynamic threshold criterion for a single particle interacting with its neighbours. Although its basis is simple, theoretical formulation of the dynamical system is essential to develop the mechanical model for a more complex system of particles. Numerical models with a continuous basis can predict accurately transport of cohesionless particles over large-scale layers of sediment as, for instance, sediment grains moving past evolutionary bedforms Ortiz and Smolarkiewicz (2009). However, prediction of critical stress conditions is often limited to local continuous assumptions (e.g. Appendix A in Ortiz and Smolarkiewicz (2006)). Instead, a discrete approach suitable for problems with internal discontinuous geometry is particularly attractive to simulate motions with scale of particle size, as in the case of initial 
and subsequent motion of bed grains.

Prevailing analytical and numerical formulations for inception of sediment motion are developed for spherical particles, see Chiew and Parker (1994), and Cheng and Chiew (1999), and Dey (2003). For instance, Ji, Munjiza, Avital, Ma, and Williams (2013), Derksen (2015) and Vowinckel, Jain, Kempe, and Fröhlich (2016) use a discrete two-way particle-fluid coupled approach to analyse the bed load transport of spherical particles in an open channel. Two way coupling must be considered for an accurate modelling of the subsequent stages of transport, particularly for suspension and saltation modes, for which flow-particles interaction is relevant. For computation of incipient motion one way coupling is justified, since in this situation motion of the particles is small and does not modify the flow substantially. This technique is used in Bravo et al. (2014), introducing extended analytical and numerical shear stress limits with a parametrical dependence on the compactness of the grains and on the bottom slope.

The assumption of spherical form is a severe constraint taking into consideration shape and orientation of grains constituting realistic configuration of beds. To consider the effect of more realistic configurations on the initiation of motion we evaluate the response of ellipsoidal particles. Ellipsoidal shape allows a broad range of forms, going from spheres to (nearly) discs, and inclination of the particle. Application of theoretical and discrete formulations to ellipsoidal particles gives a straightforward explanation of the wide band of experimental results observed by Shields (1936), by covering to a significant extent its dispersion of shapes, compactnesses and orientations. Extension of solutions to ellipsoidal forms not only benefits generalisation of threshold stress results but also aids identification of possible modes of initial particle displacement. Thereby, ellipsoidal grain starts moving by rolling or sliding but a third case can be recognised when grain detaches from one contact and slides over other contact point. This sliding mode can be dominant in all regimes for flaky particles at moderate angles of inclination (Section 4).

In this paper we formulate an analytical solution and a discrete approach to establish the dynamic conditions for the initiation of motion of an inclined ellipsoidal particle, resting on a bottom row of unmoving cylinders, and for laminar and turbulent regimes. Particle inclination is considered in computation of drag and lift forces via a blending function between drag and lift coefficients for normal and parallel flows, along the lines of Mando and Rosendahl (2010). Theoretical solution (Section 2) distinguishes rolling and the two types of sliding as possible onsets of motion. However, strict stress limit given by analytical solution is recurrently insufficient because it is not able to represent states subsequent of starting displacement. After removal, particle can find new confined equilibrium positions without reaching sensible motion; accordingly, threshold stresses corresponding to these trapped modes must be rejected. Ability of a discrete numerical procedure to reproduce more complex configurations of either particle equilibrium or particle motion permits to identify confined modes, and to explore and define mixed modes of incipient transport, as combination of rolling and the two forms of sliding. It is observed that this mixed type of motion dominates for particles with small sphericity and large inclinations for the whole range of friction Reynolds number considered.

To compute the critical shear stress by a numerical approach, we introduce in Section 3 a solution founded on the discrete element method (see e.g. Shi and Goodman (1985) and Bravo, Pérez-Aparicio, and Gómez-Hernández (2015)). The discrete element method considers the global behaviour of the particles through the single contribution of each member and the multiple interaction of particles by friction and non-penetrating contacts. Resulting momentum balance incorporates contact restrictions among particles, and contact with the sedimentary bed modelled as an aggregate of particles (Fig. 1). The discrete method computes the critical shear stress once the particle has suffered a continuous finite displacement.

Results of the experiments are discussed in Section4. Section concludes with main results arranged as critical value of the wall shear stress in function of friction Reynolds number, resembling original Shields diagram. Previous attempts to extend Shields diagram were focused on a variety of conditions such as the experiments done on fine granular (silica and mica) solids by Mantz (1977), 
experiments on the intermediate values of particle Reynolds number by Yalin and Karahan (1979), experiments on an increased range of particle Reynolds number by Miller, Mccave, and Komar (1977), experiments with inclined beds by Mohtar and Munro (2013), or numerical experiments on variable entrainment angle and bed slope by Bravo et al. (2014). Here, enhanced diagrams include parametric dependencies on sphericity and inclination, as well as theoretical bounds for rolling and sliding modes.

\section{Analytical model}

The formulation of the theoretical model for the initiation of motion of a single ellipsoidal particle resting on the bed starts by defining the bottom layer. Bottom layer is a two dimensional layer with two rows of particles (left Fig. 1), and is established as a repetition of the pattern shown in right Fig. 1. Bottom row is constituted by unmoving cylinders (Wiberg and Smith (1985)). Cylinders are fixed by lateral constraints (represented in right Fig. 1) and have maximum compactness (and hence touch each other). A scatter of ellipsoidal particles with an initial stable orientation but able to move forms the top layer. Lower row partially restricts motion of the upper grains and determines the roughness of the bed surface. Flow is planar, major axis and minor axis of the ellipsoid of revolution belong to the flow plane, and consequently particle motion is restricted to the flow plane. Nevertheless, computation of fluid-particle forces and weight requires complete three dimensional geometry.

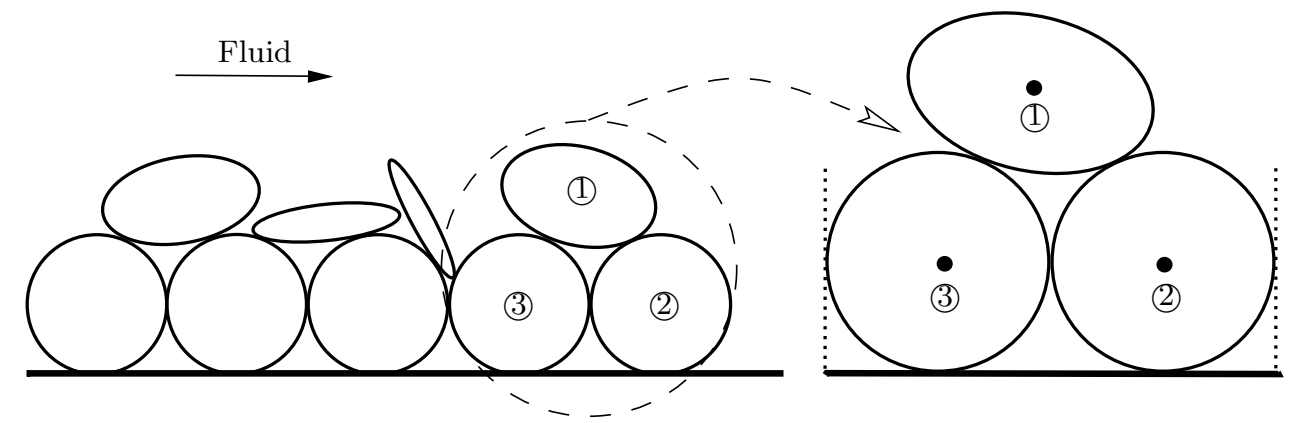

Figure 1 Sediment bed composed by the repetition of a simple pattern of cylinders and a series of ellipsoids on the top with stable orientation (right)

The geometry of the bed is specified by the radius $R$ of the cylinders, by the semi-axes of the ellipsoid, $a, b=a f_{1}$, and $c=a f_{2}$, and by $\alpha$, angle of inclination of the ellipsoidal particle (see Fig. 2). We restrict our study to ellipsoids of revolution, hence $f_{1}=f_{2}=f$. Rest of necessary geometrical variables depicted in Fig. 2 -entrainment angles $\beta_{12}, \beta_{13}$, angles $\gamma_{12}, \gamma_{13}$, and distances $d_{12}, d_{13}$-are elucidated in the Appendix.

The ellipsoidal particle initiates its motion by sliding or by rolling. In the sliding mode, particle moves while maintaining contact with the two neighbouring cylinders is maintained, giving rise to maximum value of friction force. Instead, rolling mode keeps one contact pivoting point between the corresponding two bodies. A third case of onset is possible. In this case, the particle detaches from one contact while slides over the other cylinder (see a sketch in Fig. 3). Top Fig. 2 depicts geometry and forces acting on an inclined particle submerged in an horizontal flow before the initiation of motion by any of the three modes. Pitching moment is not considered since uniform ambient wind has not side component and symmetry about this axis is assumed. 

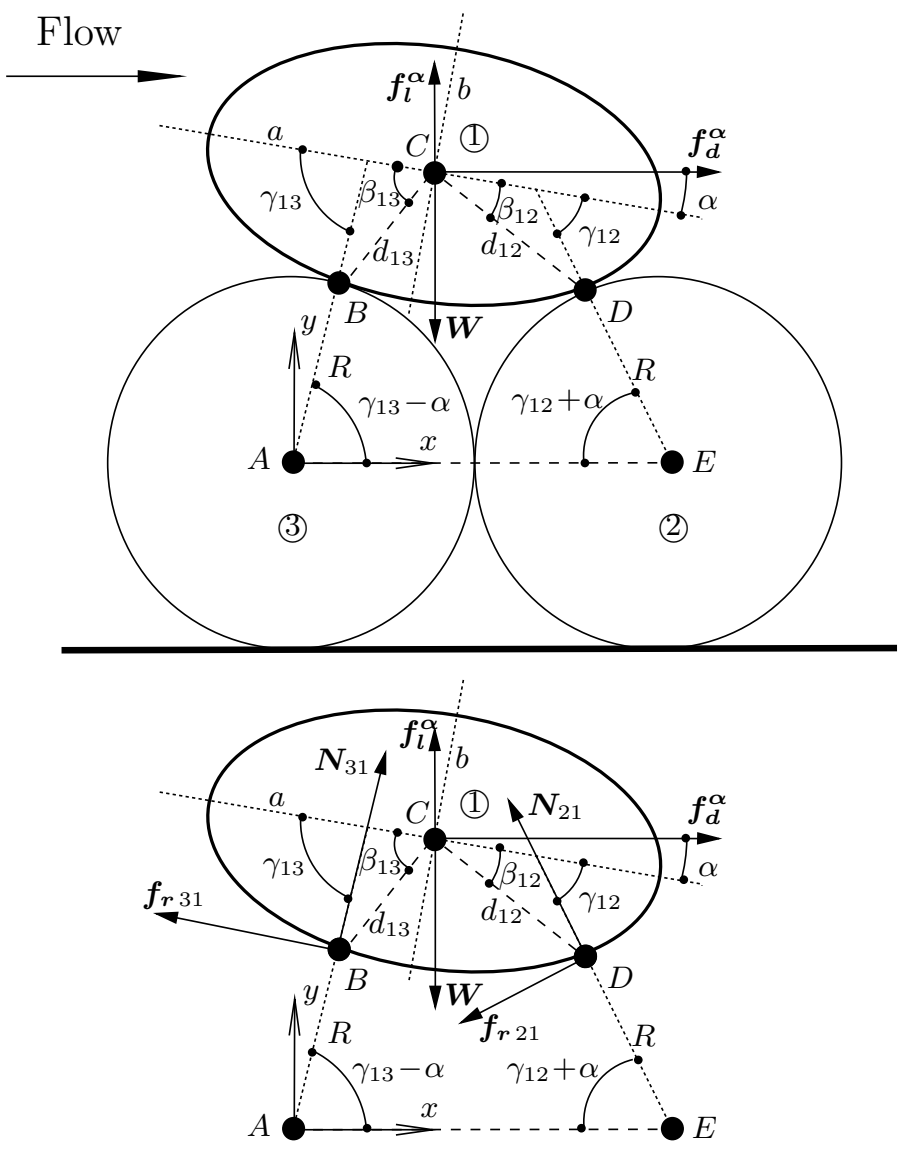

Figure 2 Forces acting on particle (top), submerged weight $\boldsymbol{W}$, drag force $\boldsymbol{f}_{d}^{\alpha}$, lift force $\boldsymbol{f}_{l}^{\alpha}$, (bottom) normal contact forces $\left|\boldsymbol{N}_{21}\right|,\left|\boldsymbol{N}_{31}\right|$ and friction contact forces $\left|\boldsymbol{f}_{\boldsymbol{r} 21}\right|$ and $\left|\boldsymbol{f}_{\boldsymbol{r} 31}\right|$.

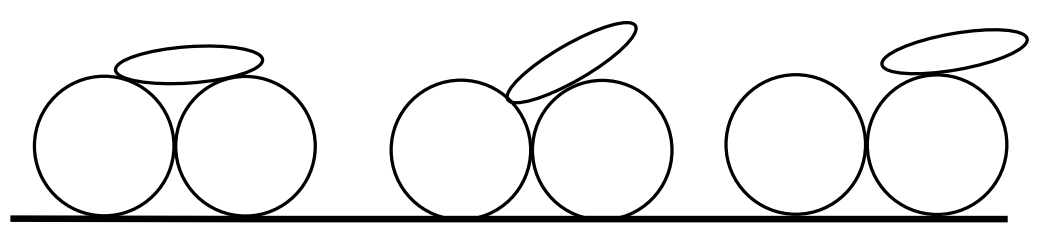

Figure 3 Initiation of motion by sliding with two contacts, $s$ (left), limit situation (center) and detachment and initiation of motion by sliding "special case", sc (right).

In case of sliding, balance equations are

$$
\begin{array}{cl}
\sum f_{x}=0 \rightarrow \quad & \left|\boldsymbol{f}_{\boldsymbol{d} \boldsymbol{s}}^{\boldsymbol{\alpha}}\right|-\left|\boldsymbol{N}_{21}\right| \cos \left(\gamma_{12}+\alpha\right)-\left|\boldsymbol{f}_{\boldsymbol{r} 21}\right| \sin \left(\gamma_{12}+\alpha\right) \\
& +\left|\boldsymbol{N}_{31}\right| \cos \left(\gamma_{13}-\alpha\right)-\left|\boldsymbol{f}_{\boldsymbol{r} 31}\right| \sin \left(\gamma_{13}-\alpha\right)=0 \\
\sum f_{y}=0 \rightarrow & \left|\boldsymbol{f}_{\boldsymbol{l} \boldsymbol{s}}^{\boldsymbol{\alpha}}\right|-|\boldsymbol{W}|+\left|\boldsymbol{N}_{21}\right| \sin \left(\gamma_{12}+\alpha\right)-\left|\boldsymbol{f}_{\boldsymbol{r} 21}\right| \cos \left(\gamma_{12}+\alpha\right) \\
& +\left|\boldsymbol{N}_{31}\right| \sin \left(\gamma_{13}-\alpha\right)+\left|\boldsymbol{f}_{\boldsymbol{r} 31}\right| \cos \left(\gamma_{13}-\alpha\right)=0, \\
\sum M=0 \rightarrow & \left|\boldsymbol{N}_{21}\right| d_{12} \sin \left(\gamma_{12}-\beta_{12}\right)-\left|\boldsymbol{f}_{\boldsymbol{r} 21}\right| d_{12} \cos \left(\gamma_{12}-\beta_{12}\right) \\
& -\left|\boldsymbol{N}_{31}\right| d_{13} \sin \left(\gamma_{13}-\beta_{13}\right)-\left|\boldsymbol{f}_{\boldsymbol{r} 31}\right| d_{13} \cos \left(\gamma_{13}-\beta_{13}\right)=0
\end{array}
$$

considering a Cartesian coordinate system $(x, y)$ such that $x$ (horizontal) positive axis is aligned with the flow direction. $\sum f_{x}, \sum f_{y}$, and $\sum M$ denote the summation of $x$ components of forces, 
the summation of $y$ components of forces and the summation of moments of all applied forces about the centre $C$ of the ellipsoid (Fig. 2), respectively. In Eqs. (1), $\boldsymbol{f}_{\boldsymbol{d} \boldsymbol{s}}^{\boldsymbol{\alpha}}$ is the drag force and $\boldsymbol{f}_{l s}^{\alpha}$ is the lift force, suffix $s$ for drag and lift forces indicates sliding. $\left|\boldsymbol{f}_{\boldsymbol{r} 21}\right|=\tan \psi\left|\boldsymbol{N}_{21}\right|$ and $\left|\boldsymbol{f}_{\boldsymbol{r}_{31} \mid}\right|=\tan \psi\left|\boldsymbol{N}_{31}\right|$ are the sliding friction Coulomb forces between particles 2 and 1 and between particles 3 and 1, respectively; $\left|\boldsymbol{N}_{21}\right|,\left|\boldsymbol{N}_{31}\right|$ are the forces normal to the surface of the particle due to contact between particles 2 and 1 and between particles 3 and 1 respectively, $|\boldsymbol{W}|=\Omega\left(\rho_{\text {sol }}-\rho_{f}\right) g$ is the submerged weight of the ellipsoid, $\Omega=\frac{4}{3} \pi(a b c)$ is the volume of the ellipsoid, and $\psi$ is the friction angle; $\rho_{\text {sol }}$ and $\rho_{f}$ are the densities of the solid and fluid respectively, and $g=|\boldsymbol{g}|$, where $\boldsymbol{g}$ is the acceleration of gravity. $\alpha$ is the angle defining particle inclination, while angles $\beta_{12}, \beta_{13}$, $\gamma_{12}, \gamma_{13}$ and distances $d_{12}, d_{13}$ gives the position of contact points $B$ and $D$, and the orientations and mechanical arms of friction and normal forces. Points of application of drag and lift forces are close to the centre of gravity (e.g. Mando and Rosendahl (2010) estimate a distance smaller than $0.07 a)$; hence moments of these forces are neglected.

Drag force and lift force can be written explicitly. For instance, drag force in terms of lift force, weight, and geometrical parameters is

$$
\left|f_{d s}^{\alpha}\right|=\frac{\left(\left|f_{l s}^{\alpha}\right|-|\boldsymbol{W}|\right)(\mathcal{A}+\mathcal{B})}{\mathcal{C}+\mathcal{D}}
$$

where

$$
\begin{aligned}
\mathcal{A}= & -2 d_{12} \sin \left(\alpha-\beta_{12}+\gamma_{12}-\gamma_{13}-2 \psi\right)+2 d_{12} \sin \left(\alpha+\beta_{12}-\gamma_{12}-\gamma_{13}\right) \\
& -d_{13} \sin \left(\alpha+\beta_{13}+\gamma_{12}-\gamma_{13}-2 \psi\right)+d_{13} \sin \left(\alpha-\beta_{13}+\gamma_{12}+\gamma_{13}-2 \psi\right) \\
& -d_{13} \cos \left(\alpha+\beta_{13}+\gamma_{12}-\gamma_{13}-2 \psi\right)+d_{13} \cos \left(\alpha-\beta_{13}+\gamma_{12}+\gamma_{13}-2 \psi\right), \\
\mathcal{B}= & d_{13} \sin \left(\alpha+\beta_{13}+\gamma_{12}-\gamma_{13}\right)+d_{13} \sin \left(\alpha-\beta_{13}+\gamma_{12}+\gamma_{13}\right) \\
& +d_{13} \cos \left(\alpha+\beta_{13}+\gamma_{12}-\gamma_{13}\right)-d_{13} \cos \left(\alpha-\beta_{13}+\gamma_{12}+\gamma_{13}\right), \\
\mathcal{C}= & -2 d_{12} \cos \left(\alpha-\beta_{12}+\gamma_{12}-\gamma_{13}-2 \psi\right)+2 d_{12} \cos \left(\alpha+\beta_{12}-\gamma_{12}-\gamma_{13}\right) \\
& +d_{13} \sin \left(\alpha+\beta_{13}+\gamma_{12}-\gamma_{13}-2 \psi\right)-d_{13} \sin \left(\alpha-\beta_{13}+\gamma_{12}+\gamma_{13}-2 \psi\right) \\
& -d_{13} \cos \left(\alpha+\beta_{13}+\gamma_{12}-\gamma_{13}-2 \psi\right)+d_{13} \cos \left(\alpha-\beta_{13}+\gamma_{12}+\gamma_{13}-2 \psi\right), \\
\mathcal{D}= & -d_{13} \sin \left(\alpha+\beta_{13}+\gamma_{12}-\gamma_{13}\right)+d_{13} \sin \left(\alpha-\beta_{13}+\gamma_{12}+\gamma_{13}\right) \\
& -d_{13} \cos \left(\alpha+\beta_{13}+\gamma_{12}-\gamma_{13}\right)+d_{13} \cos \left(\alpha-\beta_{13}+\gamma_{12}+\gamma_{13}\right),
\end{aligned}
$$

or, in concise form

$$
\left|f_{d s}^{\alpha}\right|=\left(|\boldsymbol{W}|-\left|f_{l s}^{\alpha}\right|\right) K_{1}
$$

where $K_{1}=-(\mathcal{A}+\mathcal{B}) /(\mathcal{C}+\mathcal{D})$ is a parameter containing all the geometric data of the ellipsoid resting on two cylinders.

In rolling, particle rotates respect to point $D$ and detaches from point $B$ (Fig. 2); therefore, $\left|\boldsymbol{N}_{31}\right|=\left|\boldsymbol{f}_{\boldsymbol{r} 31}\right|=0$. Balance equations for rolling are

$$
\begin{aligned}
& \sum f_{x}=0 \rightarrow\left|\boldsymbol{f}_{\boldsymbol{d} \boldsymbol{r}}^{\boldsymbol{\alpha}}\right|-\left|\boldsymbol{N}_{21}\right| \cos \left(\gamma_{12}+\alpha\right)-\left|\boldsymbol{f}_{\boldsymbol{r} 21}\right| \sin \left(\gamma_{12}+\alpha\right)=0, \\
& \sum f_{y}=0 \rightarrow\left|\boldsymbol{f}_{\boldsymbol{l} \boldsymbol{r}}^{\boldsymbol{\alpha}}\right|-|\boldsymbol{W}|+\left|\boldsymbol{N}_{21}\right| \sin \left(\gamma_{12}+\alpha\right)-\left|\boldsymbol{f}_{\boldsymbol{r} 21}\right| \cos \left(\gamma_{12}+\alpha\right)=0, \\
& \sum M=0 \rightarrow\left|\boldsymbol{N}_{21}\right| d_{12} \sin \left(\gamma_{12}-\beta_{12}\right)-\left|\boldsymbol{f}_{\boldsymbol{r} 21}\right| d_{12} \cos \left(\gamma_{12}-\beta_{12}\right)=0,
\end{aligned}
$$

where now subscript $r$ in drag force and lift force indicates rolling, while friction force-lower than 
in sliding-is unknown.

Drag in terms of lift, weight, and geometry can be written as

$$
\left|\boldsymbol{f}_{\boldsymbol{d} \boldsymbol{r}}^{\boldsymbol{\alpha}}\right|=\frac{\left(|\boldsymbol{W}|-\left|\boldsymbol{f}_{\boldsymbol{l} \boldsymbol{r}}^{\boldsymbol{\alpha}}\right|\right)}{\tan \left(\alpha+\beta_{12}\right)}=\left(|\boldsymbol{W}|-\left|\boldsymbol{f}_{\boldsymbol{l} \boldsymbol{r}}^{\boldsymbol{\alpha}}\right|\right) K_{2}
$$

where $K_{2}=1 /\left(\tan \left(\alpha+\beta_{12}\right)\right)$ contains the geometrical data.

Third situation-denoted by subscript $s c$-gives rise to particle detachment from point $B$ and its sliding over cylinder (2) (Fig. 2). Here, balance of forces is almost identical as Eqs. (1),

$$
\begin{aligned}
& \sum f_{x}=0 \rightarrow\left|\boldsymbol{f}_{\boldsymbol{d} \boldsymbol{s c}}^{\boldsymbol{\alpha}}\right|-\left|\boldsymbol{N}_{21}\right| \cos \left(\gamma_{12}+\alpha\right)-\left|\boldsymbol{f}_{\boldsymbol{r} 21}\right| \sin \left(\gamma_{12}+\alpha\right)=0, \\
& \sum f_{y}=0 \rightarrow\left|\boldsymbol{f}_{\boldsymbol{l} \boldsymbol{s c}}^{\boldsymbol{\alpha}}\right|-|\boldsymbol{W}|+\left|\boldsymbol{N}_{21}\right| \sin \left(\gamma_{12}+\alpha\right)-\left|\boldsymbol{f}_{\boldsymbol{r} 21}\right| \cos \left(\gamma_{12}+\alpha\right)=0, \\
& \sum M=0 \rightarrow\left|\boldsymbol{N}_{21}\right| d_{12} \sin \left(\gamma_{12}-\beta_{12}\right)-\left|\boldsymbol{f}_{\boldsymbol{r} 21}\right| d_{12} \cos \left(\gamma_{12}-\beta_{12}\right)=0
\end{aligned}
$$

with (maximum) sliding friction Coulomb friction force $\left|\boldsymbol{f}_{\boldsymbol{r} 21}\right|=\left|\boldsymbol{N}_{21}\right| \tan \psi$.

By recasting Eqs.(6), drag force is expressed in analogous form as Eqs. (3) and (5),

$$
\left|\boldsymbol{f}_{\boldsymbol{d} \boldsymbol{s c}}^{\alpha}\right|=-\frac{\left(\left|\boldsymbol{f}_{\boldsymbol{l s c}}^{\alpha}\right|-|\boldsymbol{W}|\right)}{\tan \left(\alpha+\gamma_{12}-\psi\right)}=\left(|\boldsymbol{W}|-\left|\boldsymbol{f}_{\boldsymbol{l} \boldsymbol{s c}}^{\alpha}\right|\right) K_{3}
$$

where $K_{3}=1 /\left(\tan \left(\alpha+\gamma_{12}-\psi\right)\right.$.

Initiation of motion occurs when force $\left|f_{d-l}^{\alpha}\right|=\left|f_{d}^{\alpha}+f_{l}^{\alpha}\right|$ satisfies either Eqs. (1) or Eqs. (4) or Eqs. (6) (or two/three of the systems simultaneously), such that

$$
\left|f_{d-l}^{\alpha}\right|=\min \left(\left|f_{d s}^{\alpha}+f_{l s}^{\alpha}\right|,\left|f_{d r}^{\alpha}+f_{l r}^{\alpha}\right|,\left|f_{d s c}^{\alpha}+f_{l s c}^{\alpha}\right|\right)
$$

Drag and lift forces are given by

$$
\left|\boldsymbol{f}_{\boldsymbol{d}}^{\boldsymbol{\alpha}}\right|=\frac{C_{d}^{\alpha}}{2} S_{e p} \rho_{f}\left(U_{f}^{\alpha}\right)^{2}, \quad\left|\boldsymbol{f}_{l}^{\alpha}\right|=\frac{C_{l}^{\alpha}}{2} S_{e p} \rho_{f}\left(U_{f}^{\alpha}\right)^{2},
$$

where $C_{d}^{\alpha}$ and $C_{l}^{\alpha}$ are the drag and lift coefficients, respectively, for an ellipsoid with inclination $\alpha$; $S_{e p}=\frac{\pi}{4} \varnothing_{e q}^{2}$ is the projected surface of the equivalent sphere of diameter $\varnothing_{e q}=\left(\frac{6 \Omega}{\pi}\right)^{1 / 3}$, and $U_{f}^{\alpha}$ is the value of mean velocity ( $x$ direction) around the inclined grain. Notation in Eqs. (9) highlights the dependence of mean velocity, drag coefficient, and lift coefficient on the particle inclination. To consider the particle inclination in the computation of drag, we use a blending function between drag coefficient for flow normal and for flow parallel to the major axis of the ellipsoidal particle, see Mando and Rosendahl (2010),

$$
C_{d}^{\alpha}=C_{d}^{0^{\circ}}+\left(C_{d}^{90^{\circ}}-C_{d}^{0^{\circ}}\right) \sin ^{3} \alpha
$$

To evaluate the drag coefficients for $\alpha=0^{\circ}$ and $\alpha=90^{\circ}$ in Eq. (10), we apply the correlation formula 
proposed by Holzer and Sommerfeld (2008) (see Eq. (9) therein), resulting in

$$
\begin{aligned}
& C_{d}^{0^{\circ}}=\frac{8}{R e} \frac{1}{\sqrt{\Phi_{l}}}+\frac{16}{R e} \frac{1}{\sqrt{\Phi}}+\frac{3}{\sqrt{R e}} \frac{1}{\Phi^{3 / 4}}+0.42 \cdot 10^{0.4(-\log \Phi)^{0.2}} \frac{1}{\Phi_{c}} \\
& C_{d}^{90^{\circ}}=\frac{8}{R e} \frac{1}{\sqrt{\Phi_{c}}}+\frac{16}{R e} \frac{1}{\sqrt{\Phi}}+\frac{3}{\sqrt{R e}} \frac{1}{\Phi^{3 / 4}}+0.42 \cdot 10^{0.4(-\log \Phi)^{0.2}} \frac{1}{\Phi_{l}}
\end{aligned}
$$

Here, $\Phi=S_{e q} / S, \Phi_{l}=S_{e p} /\left(S / 2-S_{l p}\right)$, and $\Phi_{c}=S_{e p} / S_{t p}$ are the sphericity, the lengthwise sphericity, and the crosswise sphericity, respectively; $S_{e q}=\pi \varnothing_{e q}^{2}$ is the surface of the equivalent sphere, $S$ is the surface of the ellipsoid, calculated by the approximate formula of Klamkin (1971), ${ }^{1}, S_{l p}=\pi a c$ is the surface obtained by the longitudinal projection of the surface of the ellipsoid, and $S_{t p}=\pi b c$ is the surface obtained by the transverse projection of the surface of the ellipsoid.

Computation of lift coefficient (see Mando and Rosendahl (2010)), is given by

$$
C_{l}^{\alpha}=C_{d}^{\alpha} \sin ^{2} \alpha \cos \alpha
$$

The formula results in zero lift-as in symmetrical particles-for $\alpha=0$ and for $\alpha=90^{\circ}$. Dependence on the flow of drag and lift coefficients is incorporated in Eqs. (11) by means of the Reynolds number of the flow over the equivalent sphere, $R e=\rho_{f} U_{f}^{\alpha} \varnothing_{e q} / \nu$, where $\nu$ is the dynamic viscosity of the fluid.

\subsection{Velocity field and threshold stress}

We establish a nondimensional form of the critical shear stress $\tau$ as a function of friction Reynolds number Re $\mathrm{e}^{\star}$ resembling a diagram of Shields (1936), but for an ellipsoidal particle. Thus nondimensional critical stress is defined as $\tau^{\star}=\tau /\left(\left(\rho_{\text {sol }}-\rho_{f}\right) g \varnothing_{e q}\right)=u^{\star 2} \rho_{f} /\left(\left(\rho_{\text {sol }}-\rho_{f}\right) g \varnothing_{e q}\right)$, where

$u^{\star}=\sqrt{\tau / \rho_{f}}$ is the friction velocity, and friction Reynolds number corresponds to the equivalent sphere, $\operatorname{Re}^{\star}=\rho_{f} u^{\star} \varnothing_{e q} / \nu$. As a result of the general geometrical and dynamical bases determined in previous Section, limiting conditions can be expanded into a wide variety of sphericities and inclinations of the ellipsoidal particles. Sequence of calculation is as follows. We compute the mean velocity $U_{f}^{\alpha}$ for a prescribed flow and geometry of the particle, and for low and high friction Reynolds number regimes (as detailed below). Mean velocity determines drag and lift forces, according to Eqs. (9). By inserting Eqs. (9) into conditions given by Eqs. (3), (5), and (7), these conditions can be concisely rewritten as

$$
\frac{C_{d}^{\alpha}}{2} S_{e p} \rho_{f} U_{f}^{\alpha 2}=\left(|\boldsymbol{W}|-\frac{C_{l}^{\alpha}}{2} S_{e p} \rho_{f} U_{f}^{\alpha 2}\right) K_{i} ; \quad i=1,2,3
$$

where $i=1$ for rolling mode and $i=2,3$ for sliding modes. Equations (13) constitute a system of six equations, three equations for laminar regime and three equations for turbulent regime. The lowest value of stress solution of the system corresponds to the relation $\tau^{\star}-\operatorname{Re}^{\star}$ for initiation of motion.

Mean velocity is computed as the average flow velocity over the area of an ellipse, obtained as the projection of the ellipsoid on a normal plane $(y, z)$ to the flow direction (see Figs. 4, 5 and 6). Hence,

$$
U_{f}^{\alpha}=\frac{1}{S_{p}^{\alpha}} \int_{S_{p}^{\alpha}} u(y, z) d S^{\prime}
$$

$\overline{{ }^{1} \text { Surface of the ellipsoid: } S} \approx 4 \pi\left(\left(a^{p} b^{p}+a^{p} c^{p}+b^{p} c^{p}\right) / 3\right)^{1 / p} ; p=1.6075$. 
where $u(y, z)$ is the flow velocity distribution and $z$ is the coordinate normal to the plane $(x, y)$; $S_{p}^{\alpha}=\pi b p_{y} / 2$ is the area of the ellipse, $p_{y}$ is the value of the length of its semi-axis in the $y$ direction, $d S^{\prime}=2 z(y) d y$ and

$$
z(y)=\sqrt{b^{2}\left(1-\frac{4\left(y-p_{y} / 2\right)^{2}}{p_{y}^{2}}\right)} .
$$

By simple geometrical considerations (see Appendix), $p_{y}$ can be computed as

$$
\frac{p_{y}}{a}=2\left[\cos \left(\arctan \left(\frac{f}{\tan \alpha}\right)\right) \sin \alpha+f \sin \left(\arctan \left(\frac{f}{\tan \alpha}\right)\right) \cos \alpha\right] .
$$

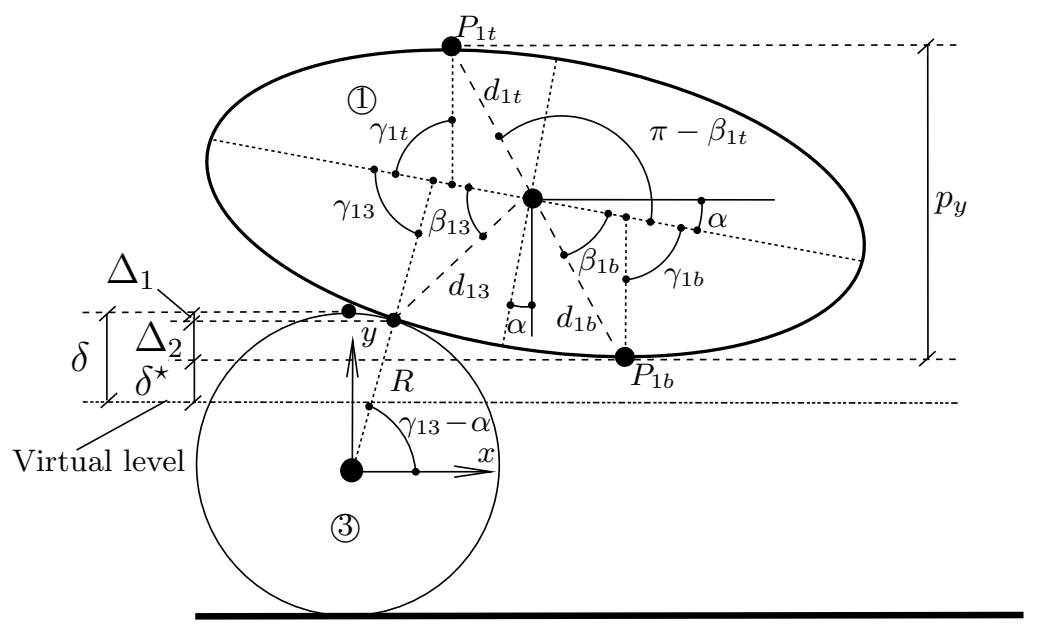

Figure 4 Vertical projection $p_{y}$, position of the virtual level $\delta$ and position $\delta^{\star}$ of the bottom of the particle respect to $\delta$. Horizontal tangents defined by the highest and lowest points (thick points) of the ellipse.

Limits of integration for Eq. (14) are from $y=\delta^{\star}$ to $y=\delta^{\star}+p_{y}$ (see Figs. 4 and 5 and Dey, Sarker, and Debnath (1999)), where $\delta^{\star}$ is the distance between the virtual level (zero velocity level) and the lowest point of the ellipsoid; $\delta^{\star}$ is calculated as

$$
\delta^{\star}=\underbrace{\xi 2 R}_{\delta}-\underbrace{R\left(1-\sin \left(\gamma_{13}-\alpha\right)\right)}_{\Delta_{1}}-\underbrace{\left(d_{1 b} \sin \left(\beta_{1 b}+\alpha\right)-d_{13} \sin \left(\beta_{13}-\alpha\right)\right)}_{\Delta_{2}},
$$

where $\delta$ is the distance from the top level of the particles on the bed to the virtual level (see thorough geometrical description in Fig. 4). Following Rijn (1984), $\delta=\xi 2 R$ and $\xi=0.25$.

For low Reynolds number (about $\operatorname{Re}^{\star}<1$ ) laminar flow conditions result in linear velocity distribution around the particle (see Fig. 5),

$$
\frac{u(y, z)}{u^{\star}}=\frac{y u^{\star}}{\nu}
$$

with mean velocity

$$
U_{f}^{\alpha}=\frac{1}{S_{p}^{\alpha}} \int_{S^{\prime}} u(y, z) d S^{\prime}=\frac{1}{S_{p}^{\alpha}} \int_{y=\delta^{\star}}^{y=\delta^{\star}+p_{y}} \frac{y u^{\star 2}}{\nu} 2 z(y) d y=\frac{p_{y} u^{\star}}{\nu} .
$$




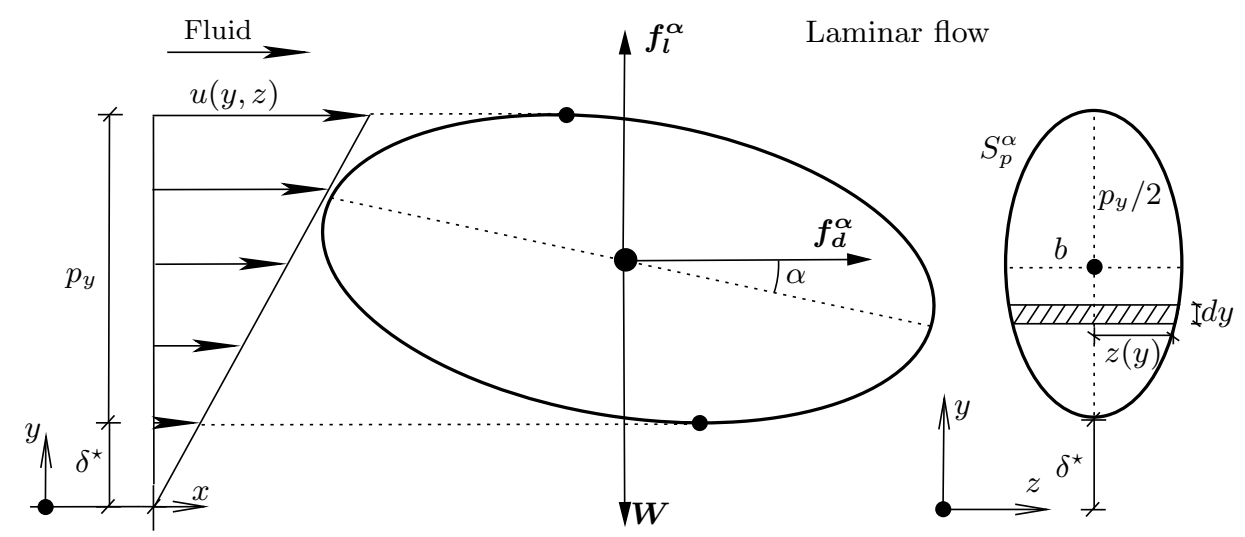

Figure 5 Velocity distribution for low particle Reynolds number.

For large particle Reynolds number $\left(\operatorname{Re}^{\star} \geq 30\right)$ velocity distribution around the particle (Fig. 6) is

$$
\frac{u(y, z)}{u^{\star}}=\frac{1}{\kappa} \log \frac{y}{y_{0}} \approx 2.5 \log \frac{y}{y_{0}},
$$

where $\kappa=0.41$ is the von Karman constant and $y_{0}$ is an equivalent bed roughness length. Typical range of bed roughness length is $y_{0} \in[2 R / 30,2 R / 10]$, see Rijn (1984).

Mean velocity is

$$
U_{f}^{\alpha}=\frac{1}{S_{p}^{\alpha}} \int_{S^{\prime}} u(y, z) d S^{\prime}=\frac{1}{S_{p}^{\alpha}} \int_{S^{\prime}} \frac{u^{\star}}{\kappa} \log \left(\frac{y}{y_{0}}\right) d S^{\prime}=\frac{1}{S_{p}^{\alpha}} \int_{y=\varepsilon}^{y=\delta^{\star}+p_{y}} \frac{u^{\star}}{\kappa} \log \left(\frac{y}{y_{0}}\right) 2 z(y) d y
$$

where $\varepsilon=y_{0}$ if $\delta^{\star}<y_{0}$ (left Fig. 6), or $\varepsilon=\delta^{\star}$ if $\delta^{\star} \geq y_{0}$ (right Fig. 6). The integral is evaluated numerically, since there is not an explicit expression.

\section{Discrete element method}

Analytical solution determines conditions of initial particle motion, but it is unable to reproduce displacements subsequent to the precise initiation. Before the potential occurrence of saltation or suspension modes, the moving particle can still be in contact with neighbouring grains (see Fig. 3 and comments on it in Section 4). To describe these incipient motions and their consequent multiple contacts and evolutionary forces we apply a discrete element procedure.

Consider two bodies $i$ and $k$. To prescribe the condition of non-penetration for the two bodies we define the constraint

$$
g_{N i k}(\boldsymbol{X})=[\boldsymbol{X}-\boldsymbol{Y}(\boldsymbol{X})] \boldsymbol{n}_{\boldsymbol{i k}} \geq 0,
$$

where $\boldsymbol{X}, \boldsymbol{Y}$ designate the location of the set of points belonging to bodies $i$ and $k$ respectively, and $g_{N i k}(\boldsymbol{X})$ is a gap function such that points are either nearby to the contrary body, $g_{N}{ }_{i k}(\boldsymbol{X})>0$, or in contact, $g_{N i k}(\boldsymbol{X}) \approx 0$. Closest points of the set $\boldsymbol{X}, \boldsymbol{Y}$ define a local coordinate system where $\boldsymbol{n}_{\boldsymbol{i k}}=-\boldsymbol{n}_{\boldsymbol{k} \boldsymbol{i}}$ is the normal unit vector and $\boldsymbol{t}_{\boldsymbol{i k}}$ is the tangential unit vector at contact point (see Fig. 7 and Appendix). Motion of contact points along the tangential direction is described by the 


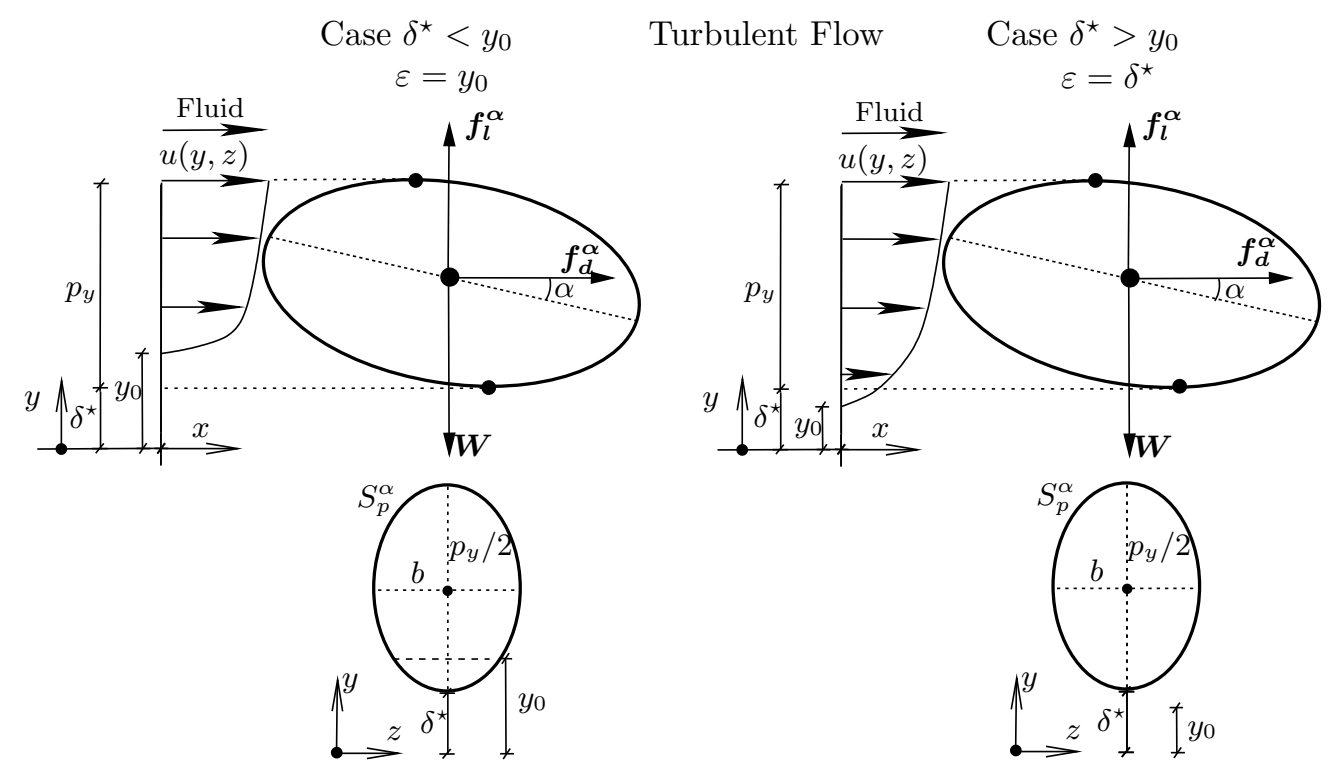

Figure 6 Velocity distribution for large particle Reynolds number.

tangential gap $g_{T i k}$ as

$$
g_{T i k}(\boldsymbol{X})=\left[\boldsymbol{X}+\boldsymbol{Q}_{\boldsymbol{i}}(\boldsymbol{X})-\boldsymbol{Y}(\boldsymbol{X})-Q_{\boldsymbol{k}}(\boldsymbol{Y}(\boldsymbol{X}))\right] \boldsymbol{t}_{\boldsymbol{i k}}
$$

where $\boldsymbol{Q}_{\boldsymbol{i}}(\boldsymbol{X})$ and $\boldsymbol{Q}_{\boldsymbol{k}}(\boldsymbol{Y}(\boldsymbol{X}))$ are the displacements of the contact points of the bodies $i$ and $k$, respectively, along the tangential direction for a given time. To specify $\boldsymbol{X}, \boldsymbol{Y}$ for ellipsoidal and spherical particles we use the algorithm given by Wellmann, Lillie, and Wriggers (2008).

Contact force $\boldsymbol{f}_{\boldsymbol{c i k}}$ imposes dynamically the non-penetration condition and is written as

$$
f_{c i k}=\left|N_{i k}\right| n_{i k}+\left|f_{r i k}\right| t_{i k}
$$

Here, $\boldsymbol{N}_{\boldsymbol{i} \boldsymbol{k}}$ and $\boldsymbol{f}_{\boldsymbol{r} \boldsymbol{i} \boldsymbol{k}}$ are the normal and tangential components of the contact force, respectively (as described in Section 2 and represented in Fig. 2). The sliding and rolling modes-symbolised in Section 2 by subindexes $r, s$ and $s c$-are distinguished by the discrete element method through the frictional Coulomb's law,

$$
\Xi_{i k}=\left|f_{r i k}\right|-\mu\left|N_{i k}\right|
$$

where $\mu\left|\boldsymbol{N}_{\boldsymbol{i k}}\right|$ is the force that produces the sliding (maximum friction force) and $\mu=\tan \psi$ is the friction coefficient. Sliding arises if $\Xi_{i k} \geq 0$, while rolling occurs if $\Xi_{i k}<0$.

Contact constitutive equations relate contact force and gap functions. To model constitutive equations we employ penalisation techniques (see Laursen (2002) and references therein). Penalisation techniques introduce two high stiffness elastic springs located in the contact points along the normal and tangential directions, with stiffness $K_{N}$ and $K_{T}$ respectively (see Fig. 7) and hence contact forces are $\left|\boldsymbol{N}_{i k}\right|=K_{N} g_{N i k}$ and $\left|\boldsymbol{f}_{r i k}\right|=K_{T} g_{T i k}$ (rolling), or $\boldsymbol{N}_{i k}=K_{N} g_{N i k}$ and $\left|\boldsymbol{f}_{r i k}\right|=\mu\left|\boldsymbol{N}_{i k}\right|$ (sliding). The procedure allows a little interpenetration between bodies, and requires tuning of stiffness parameters. However, the approach is efficient since it does not need additional unknowns (see Ch. 10 of Belytschko, Liu, and Moran (2000) for discussion). Hence, the method reduces computational costs for problems with large number of contacts in compare with the Lagrange multipliers technique (Belytschko and Neal (1991)). 


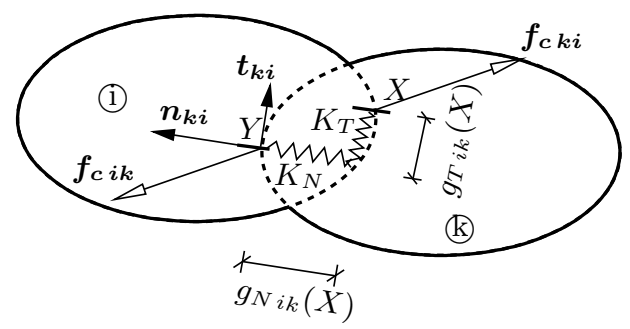

Figure 7 Contact between two bodies: $g_{N i k}$ defines the maximum penetration and $g_{T i k}$ defines the tangential displacement.

The equations of motion for a system of $n_{b d}$ interacting bodies are formulated by Hamiltonian mechanics and are solved by the discrete element method. The Equations of motion for each body are expressed via the Hamiltonian function $\mathcal{H}$ defining the total energy of the system,

$$
\begin{aligned}
& \mathcal{H}\left(\boldsymbol{Q}_{\boldsymbol{i}}(x, y, t), \boldsymbol{P}_{\boldsymbol{i}}(x, y, t)\right)=\sum_{i=1}^{n_{b d}}\left[\mathcal{K}\left(\boldsymbol{P}_{\boldsymbol{i}}(x, y, t)\right)+\mathcal{V}\left(\boldsymbol{Q}_{\boldsymbol{i}}(x, y, t)\right)\right], \\
& \mathcal{K}\left(\boldsymbol{P}_{\boldsymbol{i}}(x, y, t)\right)=\frac{1}{2} \int_{\Omega^{i}} \frac{\boldsymbol{P}_{\boldsymbol{i}}(x, y, t)^{2}}{\rho_{\text {sol }}} \mathrm{d} \Omega,
\end{aligned}
$$

where $\boldsymbol{P}_{\boldsymbol{i}}(x, y, t)$ is the linear momentum of any point at $(x, y)$ at time $t$ of body $i, \mathcal{K}\left(\boldsymbol{P}_{\boldsymbol{i}}(x, y, t)\right)$ is the kinetic energy and $\mathcal{V}\left(\boldsymbol{Q}_{\boldsymbol{i}}(x, y, t)\right)$ is the potential energy. Motion corresponds to the minimum energy of the system and is given by the solution $\left(\boldsymbol{Q}_{\boldsymbol{i}}(x, y, t), \boldsymbol{P}_{\boldsymbol{i}}(x, y, t)\right)$ of the Hamiltonian canonical equations,

$$
\dot{\boldsymbol{Q}}_{i}=\frac{\partial \mathcal{H}}{\partial \boldsymbol{P}_{\boldsymbol{i}}}=\frac{\boldsymbol{P}_{\boldsymbol{i}}}{\rho_{\text {sol }}} ; \quad \dot{\boldsymbol{P}}_{\boldsymbol{i}}=-\frac{\partial \mathcal{H}}{\partial \boldsymbol{Q}_{\boldsymbol{i}}}=-\nabla \mathcal{V}\left(\boldsymbol{Q}_{\boldsymbol{i}}\right)
$$

The method approximates Eqs. (20) by a linear discrete representation of displacements and linear momentum with nodal shape functions $\mathbf{N}_{\mathbf{i}}(x, y)$ such that for each body $i$,

$$
Q_{i}=\mathrm{N}_{\mathrm{i}} q_{i} ; P_{i}=\mathrm{N}_{\mathrm{i}} p_{i}
$$

Discrete variables $\boldsymbol{q}_{\boldsymbol{i}}$ and $\boldsymbol{p}_{\boldsymbol{i}}$ are values of displacements and momentum at the centroid of the particle. Nodal shape functions are defined as

$$
\mathbf{N}_{\mathbf{i}}(x, y)=\left[\begin{array}{rrr}
1 & 0 & -\left(y-y_{i}\right) \\
0 & 1 & \left(x-x_{i}\right)
\end{array}\right]
$$

where $\left(x_{i}, y_{i}\right)$ are the coordinates of the centroid. By replacing Eqs. (21) in Eqs. (20), we accomplish the following discrete system of equations

$$
\dot{\boldsymbol{q}}_{\boldsymbol{i}}=\boldsymbol{M}_{\boldsymbol{i}}^{-1} \boldsymbol{p}_{\boldsymbol{i}} ; \quad \dot{\boldsymbol{p}}_{\boldsymbol{i}}=\boldsymbol{f}_{\boldsymbol{c} \boldsymbol{i}}+\boldsymbol{f}_{\boldsymbol{d i}}+\boldsymbol{f}_{\boldsymbol{l i}}+\boldsymbol{W}_{\boldsymbol{i}}, i=1, \ldots, n_{b d},
$$

representing the discrete counterpart in Hamiltonian form for a system of particles of the analytical balance equations given by Eqs. (1), (4), or (6). In Eqs. (22) $\boldsymbol{M}_{\boldsymbol{i}}$ is the lumped mass matrix containing the mass of particle $i$ and its corresponding virtual mass, and $\dot{\boldsymbol{q}}_{\boldsymbol{i}}$ and $\dot{\boldsymbol{p}}_{\boldsymbol{i}}$ are the time derivatives of the displacement and linear momentum, respectively. Time integration of Eqs. (22) is performed by an implicit one step algorithm proposed by Bravo, Pérez-Aparicio, and Laursen (2012). The resultant of contact forces acting on body $i, \boldsymbol{f}_{\boldsymbol{c} \boldsymbol{i}}$, and the flow-particle forces $\boldsymbol{f}_{\boldsymbol{d} \boldsymbol{i}}$ and 
$\boldsymbol{f}_{l i}$ are updated every time step since contacts, position and orientation of the particles vary in time.

\section{Analysis}

To attempt results for realistic configurations of sedimentary beds we compute analytical and discrete solutions for a variety of inclinations, particle shapes, and friction Reynolds numbers, covering both laminar and turbulent regimes. Shape is selected by prescribing $f, \alpha$, and $a$, while Reynolds number is adjusted by rescaling its components. Initial bed layout (Fig. 1) is the same for analytical and numerical solutions. Numerical solution computes critical stresses by prescribing shear velocity, increasing its value until inception of movement is detected. Detection requires specification of a criterion to initiation of motion. Threshold is established when particle has passed its neighbour limit, hence rejecting any confined displacements (see Fig. 8).

Boundary layer flow of the fluid (air, $\rho_{f}=1 \mathrm{~kg} / \mathrm{m}^{3}$ and $\nu=10^{-5} \mathrm{~m}^{2} / \mathrm{s}$ ) is undisturbed by the moving particle; $g=9.81 \mathrm{~m} / \mathrm{s}^{2}, \rho_{\text {sol }}=2500 \mathrm{~kg} / \mathrm{m}^{3}, \psi=15^{\circ}$ between particles and $\psi=40^{\circ}$ between particles and boundaries (latter friction is augmented to resemble unmoving cylinders). Therefore the small ratio between density of air and particle permits to neglect the virtual mass force. Time increment for integration of Eqs. (22) is $\Delta t=0.0025 \mathrm{~s}$, total number of time steps is 5000 , increment of shear velocity for each calculation is $\Delta u^{\star}=0.001 \mathrm{~m} / \mathrm{s}$, bed roughness length is $y_{0}=2 R / 25$, and $K_{N}=K_{T}=10^{6} \mathrm{~N} / \mathrm{m}$. Minimum length of larger semiaxis is $a=5 \times 10^{-5} \mathrm{~m}$ to avoid ill-conditioned system of equations of the discrete solution. To simulate very small $R^{\star}$ number conditions, the fluid viscosity is increased to $\nu=10^{-3} \mathrm{~m}^{2} / \mathrm{s}$. Figures 9 and 10 constitute an extract of the series of numerical calculations to illustrate discussion of results. These figures show limiting stress for the initiation of motion in terms of particle shape-via $f$ and angle of inclination $\alpha$-for several friction Reynolds number belonging to laminar and turbulent regimes. Figures 11 and 12 plot a selection of numerical and analytical answers superimposed to scrutinise differences between both solutions. Figures 13-16 include analytical and numerical answers as modified Shields diagrams, where nondimensional critical shear stress is plot in terms of friction Reynolds number, for values of $f=1.0,0.75,0.50,0.25$ and for values of $\alpha=0^{\circ}, 15^{\circ}, 25^{\circ}, 35^{\circ}$. In the first of Fig. 13, the experimental envelopes by Shields (1936), Miller et al. (1977), Buffington and Montgomery (1997) and the experimental points of the previous reference complement the diagrams. For clarity, the rest of the figures the analytical and numerical results are only compared with the widely used experimental envelope of Shields (1936).

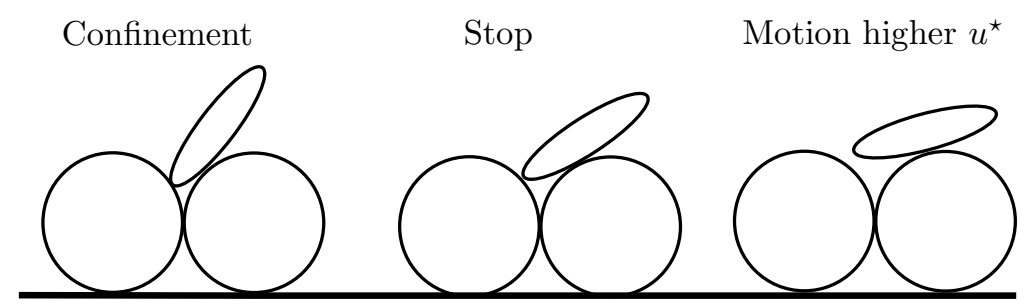

Figure 8 Initiation of motion in confined situation (left). Equilibrium in a new confined situation (centre). Continuation for higher $u^{\star}$ (right).

\subsection{Results and Discussion}

Area of the particle exposed to the flow is determinant of critical stress value. Area exposed increases once inclination increases, resulting in larger average flow velocity over the area (Eqs. (14) and (15)), and in an augmentation of drag coefficient through Eq. (10). Value of $f$ is also relevant 


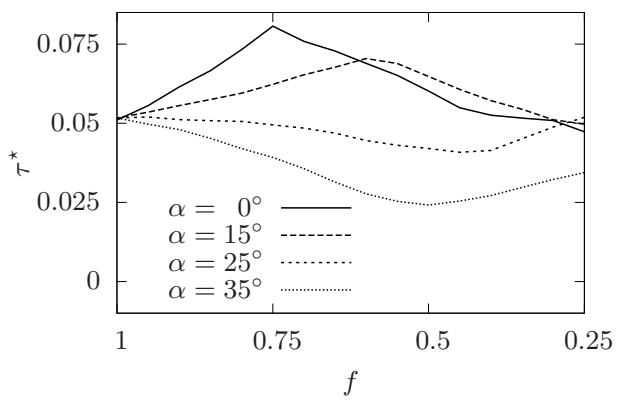

(a)

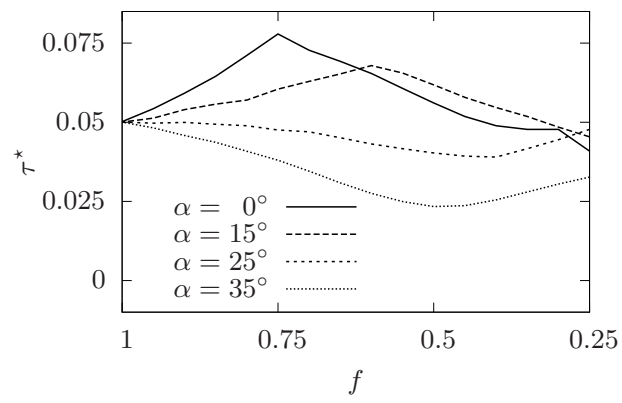

(b)

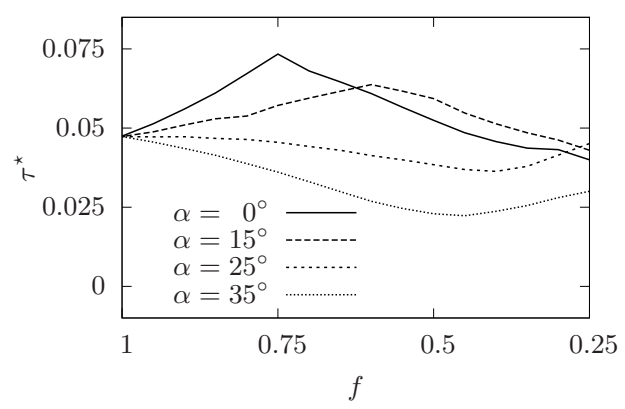

(c)

Figure 9 Critical stress in terms of $f$ for $\operatorname{Re}^{\star}=0.1,0.5,1$. Numerical results

to the growth of exposed area, particularly for the lowest range of values of $\alpha$. However, effects of $f$ and $\alpha$ are not restricted to the increase of the windswept area. Variations of shape and inclination are also accompanied by variation of mechanical arm, significant for rolling mode, and by the manifest change of location of forces.

Figure 9 plots numerical critical stress for three representative values of friction Reynolds number in the laminar range, $\operatorname{Re}^{\star}=0.1,0.5$, and 1 . For $f \gtrsim 0.75$ rolling mode determines initial motion, while for $f \lesssim 0.6$ mixed mode combining partial rolling, sliding and $s c$ sliding dominate (Table 1 shows a brief guide of critical shear stresses and an indicator of the mode). In rolling region, increase of stress for decreasing values of $f$ and for low inclinations is mainly ascribable to decrease of mechanical arm once particle becomes flaky (but not enough to prevent rolling!). Instead, higher values of inclination $\left(\alpha \gtrsim 15^{\circ}\right)$ contribute dominantly to enlarge mechanical arm and hence, contribute to a reduction of stress needed to roll the particle. A monotone decrease of critical stress with increase of angle for $f \gtrsim 0.75$ is also consistent with the corresponding increment of angle of attack, reducing the force needed to move the particle by rolling or by sliding. This consequence is manifest for high values of $\alpha$. Furthermore, for $f \geqq 0.6$ the required threshold stress diminishes with decreasing $f$ (Fig. 9). In this range, decrement of $f$ gives rise to a considerable reduction of the diameter of the equivalent sphere and hence to a considerable increase of shear velocity for a given friction Reynolds number. Ergo a reduction of critical stress value ensues from the significant growth of mean velocity (see Eq. (17) and Fig. 5).

Figure 10 plots critical shear stress in terms of $f$, for different values of $\alpha$, and for three friction Reynolds number values prototypical of turbulent range, $\operatorname{Re}^{\star}=500,1000$, and 1500 . Table 2 shows the guide of critical shear stresses and indicators of the mode. For values of $f \gtrsim 0.8$ stress behaves as in the laminar case, including the decrease of critical stress value for high values of $\alpha$. If $f \lesssim 0.8$ the origin of motion occurs by a mixed mode of rolling, sliding and $s c$ sliding, and limiting stress grows monotonically for $\alpha<25^{\circ}$, while the starting of growth for $\alpha>25^{\circ}$ takes place for $0.5<f<0.75$ (Fig. 10). This increase of demanded stress with decreasing values of $f$ comes from 
Table 1 Limiting stress for $\mathrm{Re}^{\star}=1$. $\mathrm{R}$ rolling, $\mathrm{M}$ mixed mode combining rolling, sliding, and SC sliding.Numerical results.

\begin{tabular}{lcccc}
\hline$f$ & $\alpha=0^{\circ}$ & $\alpha=15^{\circ}$ & $\alpha=25^{\circ}$ & $\alpha=35^{\circ}$ \\
\hline 1.00 & R 0.047 & R 0.047 & R 0.047 & R 0.047 \\
0.90 & R 0.055 & R 0.050 & R 0.047 & R 0.043 \\
0.80 & R 0.067 & R 0.053 & R 0.046 & R 0.038 \\
0.70 & M 0.068 & R 0.059 & R 0.044 & R 0.033 \\
0.60 & M 0.060 & R 0.063 & R 0.041 & R 0.026 \\
0.50 & M 0.052 & SC 0.059 & M 0.038 & M 0.022 \\
0.40 & M 0.045 & SC 0.051 & M 0.036 & M 0.023 \\
0.30 & M 0.043 & M 0.046 & M 0.041 & M 0.028 \\
0.25 & M 0.039 & M 0.043 & SC 0.045 & M 0.030 \\
\hline
\end{tabular}

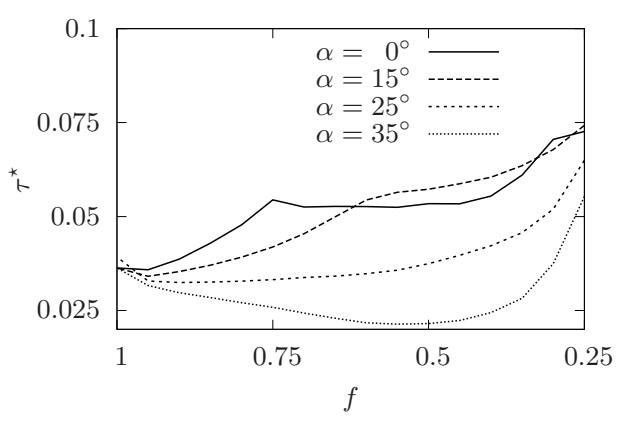

(a)

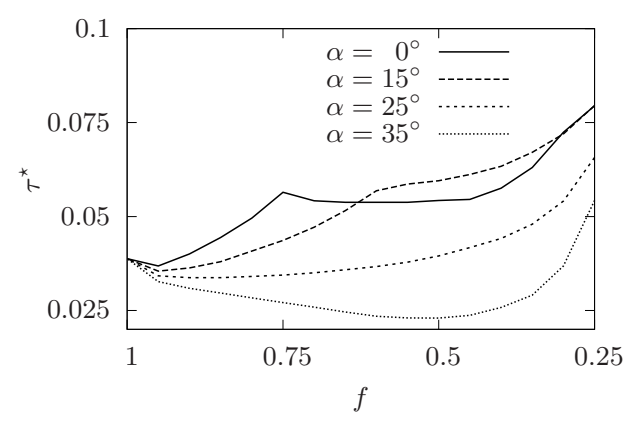

(b)

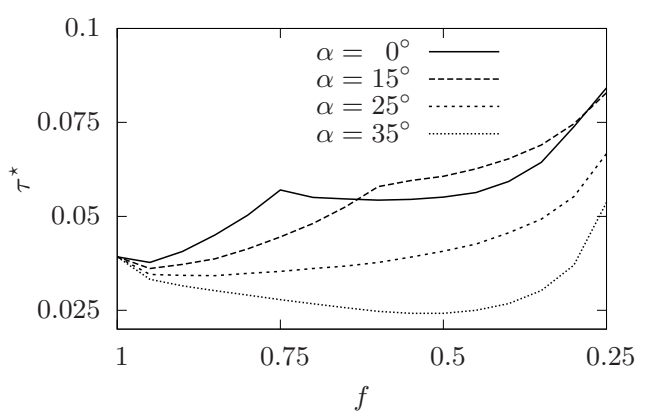

(c)

Figure 10 Critical stress in terms of $f$ for $\operatorname{Re}^{\star}=500,1000,1500$. Numerical results

the reduction of resultant applied force, attributable to the relevant reduction of exposed area and to the logarithmic distribution of velocity (see Eq. (18) and Fig. 6). Otherwise, Fig. 10 shows that in the range of low values of $f$ and high values of $\alpha$, a small increment of inclination yields to significant reduction of critical stress.

Deviations of numerical answers from analytical answers are revealed in Fig. 11 (laminar regime) and Fig. 12 (turbulent regime). Figures 11 and 12 plot the analytical limiting stress for rolling, sliding and $s c$ sliding modes along with numerical results. In the case of null or small angle of inclination, analytical solution of critical stress for rolling, sliding, and sc sliding intersect at a point, and minimum stress turns from rolling to sliding for decreasing values of $f$. Numerical solution follows analytical rolling mode for $f>0.75\left(\alpha=0^{\circ}\right)$ and for $f>0.6\left(\alpha \geq 15^{\circ}\right)$. In both analytical and numerical methods, range of rolling expands slightly to lower values of $f$ with increasing $\alpha$, since mechanical arm grows. For high inclinations, rolling region in the laminar regime extends to lower values of $f$ than turbulent, also detailed in Tables 1 and 2. In case of small value of $f$, particle starts moving by rolling and reaches an intermediate position of equilibrium; rolling puts grain 


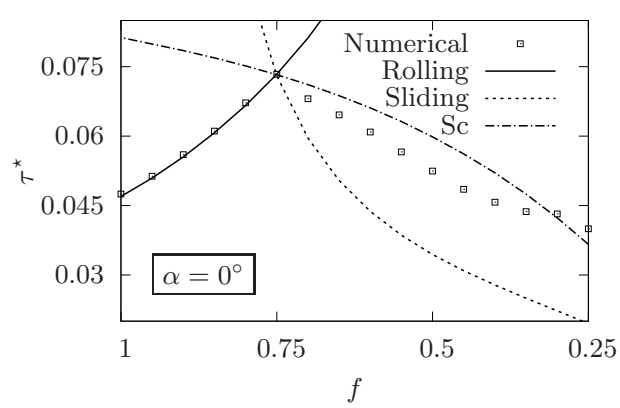

(a)

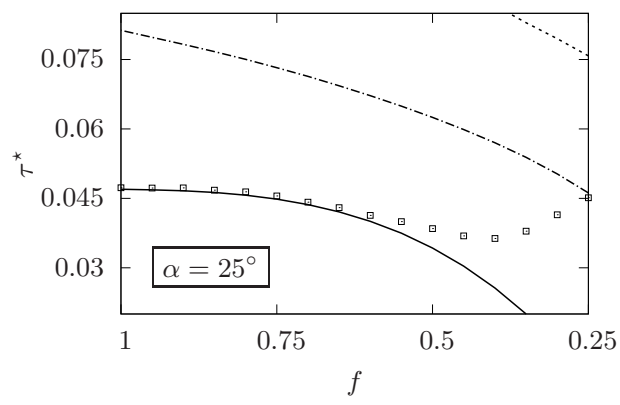

(c)

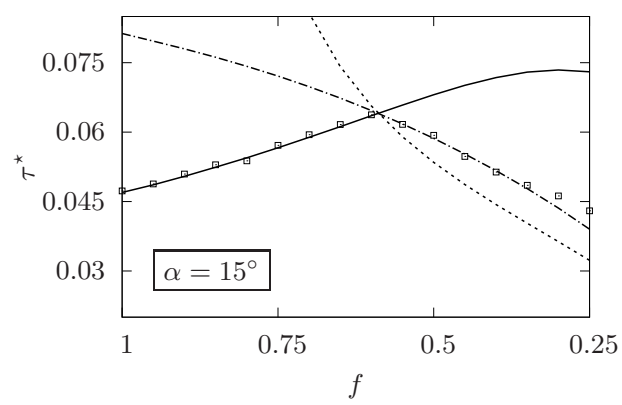

(b)

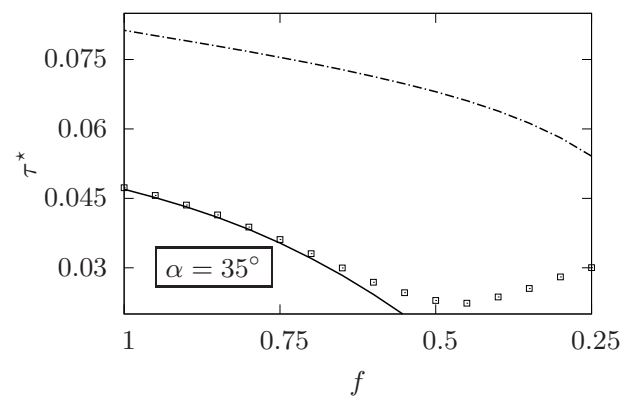

(d)

Figure 11 Critical stress $\tau^{\star}$ in terms of $f$ and $\alpha$ for $\operatorname{Re}^{\star}=1$. For $\alpha=0^{\circ}$ (a), $\alpha=15^{\circ}$ (b), $\alpha=25^{\circ}$ (c) and $\alpha=35^{\circ}$ (d)

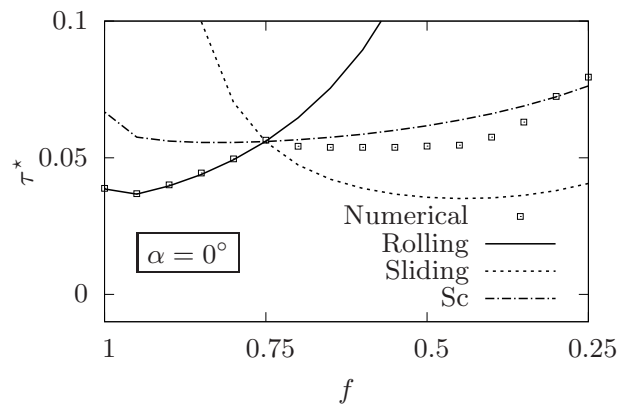

(a)

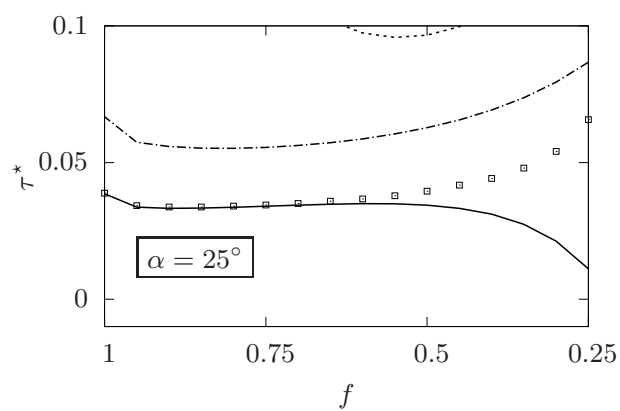

(c)

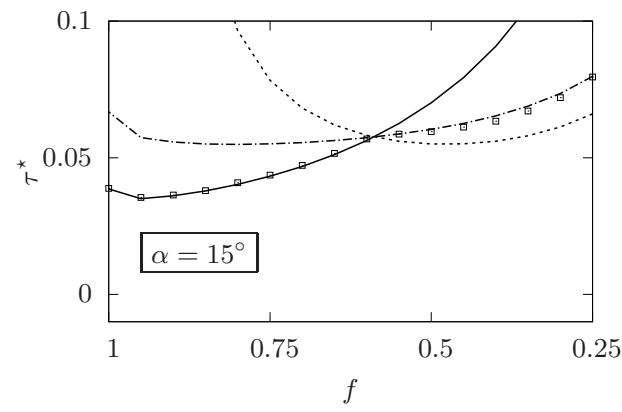

(b)

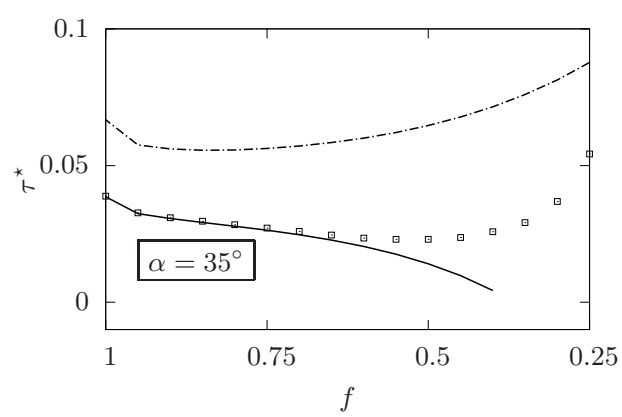

(d)

Figure 12 Critical stress $\tau^{\star}$ in terms of $f$ and $\alpha$ for $\operatorname{Re}^{\star}=1000$. For $\alpha=0^{\circ}$ (a), $\alpha=15^{\circ}$ (b), $\alpha=25^{\circ}$ (c) and $\alpha=35^{\circ}$ (d) 
Table 2 Limiting stress for $\mathrm{Re}^{\star}=1000$. $\mathrm{R}$ rolling, $\mathrm{M}$ mixed mode combining rolling, sliding, and SC sliding. Numerical results.

\begin{tabular}{lcccc}
\hline$f$ & $\alpha=0^{\circ}$ & $\alpha=15^{\circ}$ & $\alpha=25^{\circ}$ & $\alpha=35^{\circ}$ \\
\hline 1.00 & R 0.038 & R 0.038 & R 0.038 & R 0.038 \\
0.90 & R 0.040 & R 0.036 & R 0.033 & R 0.030 \\
0.80 & R 0.049 & R 0.040 & R 0.034 & R 0.028 \\
0.70 & R 0.054 & R 0.047 & R 0.035 & R 0.025 \\
0.60 & SC 0.053 & R0.056 & R 0.036 & M 0.023 \\
0.50 & M 0.054 & SC 0.059 & M 0.039 & M 0.022 \\
0.40 & M 0.057 & SC0.063 & M 0.044 & M 0.025 \\
0.30 & SC 0.072 & SC 0.071 & M 0.054 & M 0.036 \\
0.25 & M 0.079 & SC 0.079 & M 0.065 & M 0.054 \\
\hline
\end{tabular}

in a more horizontal position, reducing the exposed area (see sketch in Fig. 8). Subsequent states can not be captured by analytical solution. However, numerical simulation continues until particle passes its neighbour limit, resulting in limiting stresses higher than analytical solution. Numerical critical stress is also higher than pure sliding, and mostly lower than $s c$ sliding analytical stress value.

Apart from potential practical usage, Shields' type diagrams help to compare numerical and analytical results with experimental results. Shields' type diagrams are represented in Figs. 13 to 16 , where critical stresses $\tau^{\star}$ are plot in terms of $\operatorname{Re}^{\star}$. To cover a wide range of shapes and inclinations, each diagram is plot for $f=1.0,0.75,0.5$, and 0.25 , while inclinations are $\alpha=0^{\circ}$, $15^{\circ}, 25^{\circ}, 35^{\circ}$, for Figs.13, 14, 15, and 16, respectively. Diagrams show stresses from analytical results for each mode, from numerical results, and from experiments by Shields (1936) and their corresponding experimental extensions from Miller et al. (1977) and Buffington and Montgomery (1997) (for clarity, the last two experimental results are only exposed in the first of Fig. 13). Experimental results are depicted as several bands enclosed by two curves corresponding to the statistical limit stress values measured in the experimental cloud of points.

Two regions can be identified in Figs. 13-16, the low particle Reynolds number (left) and the high particle Reynolds number (right). Between both regions, a range defined approximately as $4<\operatorname{Re}^{\star}<30$ specifies a transition region, where the representation of drag and lift given by Eqs. (9) is not adequate due to a poor velocity profile assessment. For all orientations numerical solution is adequately close to experimental limits if particles are nearly spherical $(f \gtrsim 0.75)$. For high inclinations, discrete computation matches laboratory results up to $f \gtrsim 0.5$. Nevertheless, once particle becomes flaky discrete solution differs substantially from experiments. Experiments by Shields and their subsequent extensions by other authors were performed with nearly spherical particles, while orientation of the particle was, presumably, very difficult to control during set-up.

Similarities among stress answers are noticeable for high values of $f$ by comparing Fig. 13(a) (repeated as 14(a), 15(a), and 16(a) for clarity) with Fig. 14(b), Fig.15(b), and Fig. 16(b). For low Reynolds number numerical stress coincides with rolling analytical results, as well as for high Reynolds number. In both regimes discrete limiting stress and analytical limiting stress by rolling are below sliding and $s c$ sliding analytical modes, therefore extending characteristics shown previously to the whole range of $\mathrm{Re}^{\star}$ considered here. In case of low values of $f$, Shields type curves also overview stresses features. In turbulent range numerical results have as upper bound $s c$ sliding threshold. For small inclinations, the calculations are over analytical sliding, while for big inclinations the calculations are below analytical sliding. For laminar region, limiting stress is again close to $s c$ analytical mode for null or small values of inclination. For higher values of $\alpha$ results have as upper limit $s c$ sliding mode. It is interesting to observe the change produced in the analytical rolling mode and the analytical sliding mode for turbulent range when very flat particles get severe inclinations (see Figs. 14 (d) and 15 (d)). When inclination increases, rolling mode turns to lower values than the two possible sliding modes, caused by a significant augmentation of the 
mechanical arm and of the exposed area.

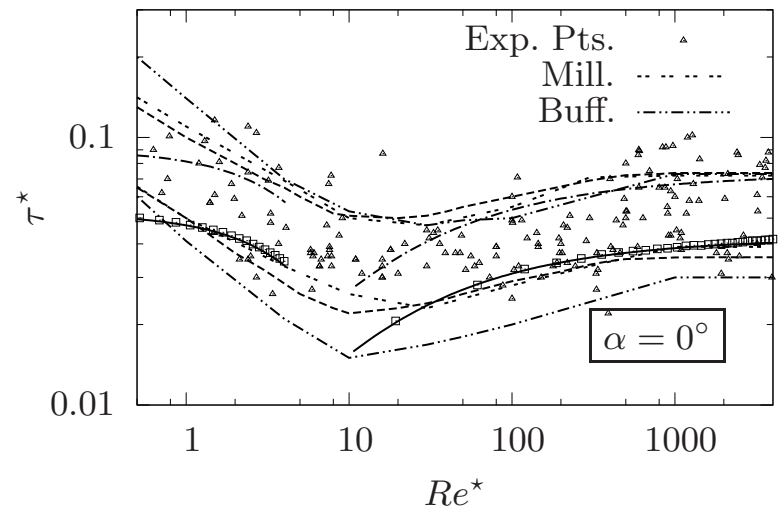

(a)

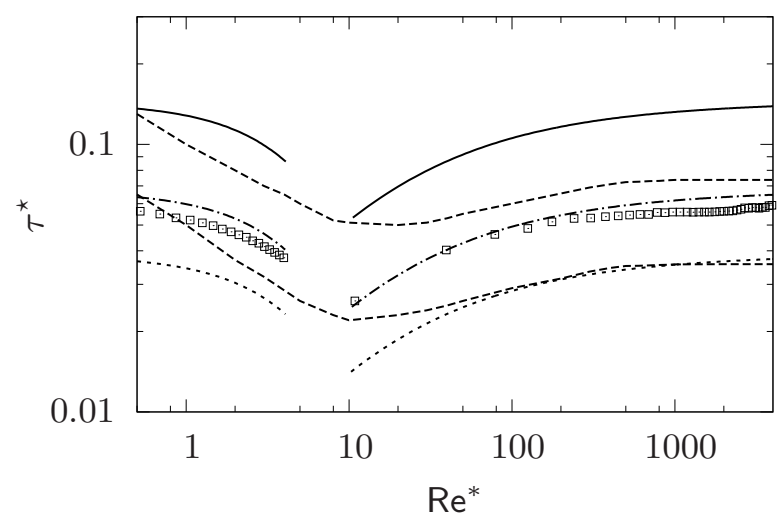

(c)

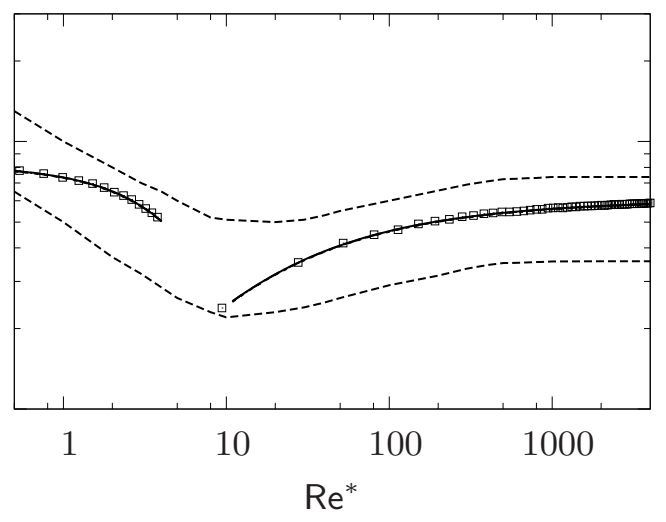

(b)

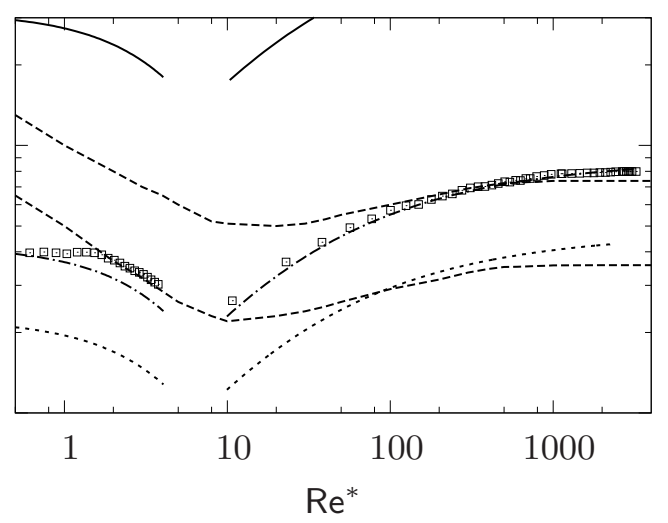

(d)

An. Rolling

An. Slid.

An. Slid."sc"-.-.-... N Num. DEM

Exp. Shields--------

Figure $13 \mathrm{Re}^{\star}, \tau^{\star}$ relation for $\alpha=0^{\circ}$ and $f=1.0,0.75,0.50,0.25$, (a), (b), (c), (d) respectively. The analytical and numerical results compared with the experimental envelopes and points from Miller et al. (1977) and Buffington and Montgomery (1997) for $f=1.0$.

\section{Conclusions}

Analytical and discrete methods were developed to study the influence of shape and inclination of ellipsoidal particles in the initiation of motion, by assessing threshold shear stress and mode of movement. Both methods consider realistic beds by including particle micromechanics based on contact and friction. Interaction with flow is simulated by drag and lift forces, taking into account ellipticity and inclination of the particles.

Computed stresses fall in the band of results by Shields, consistent with the wide variety of shape and inclination of real particles employed in the experiments. Analytical approach is essential to understand underlying physics of the incipient motion. It is too restricted, since it models the breakage of the equilibrium without taking into account the subsequent movements. Discrete approach is a necessary complement to model the initiation and subsequent stages of particle motion, circumventing the limitations of analytical at these complex configurations, where trapped positions are frequent. Hence, discrete method assures the completion of the criterion of motion 


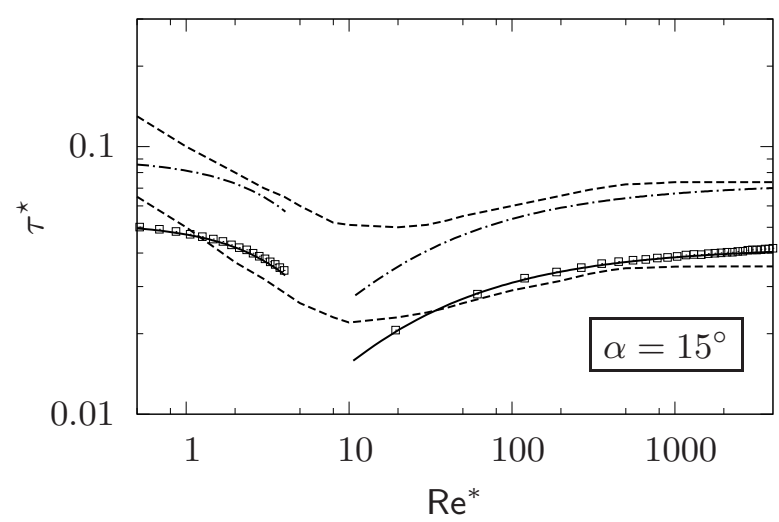

(a)

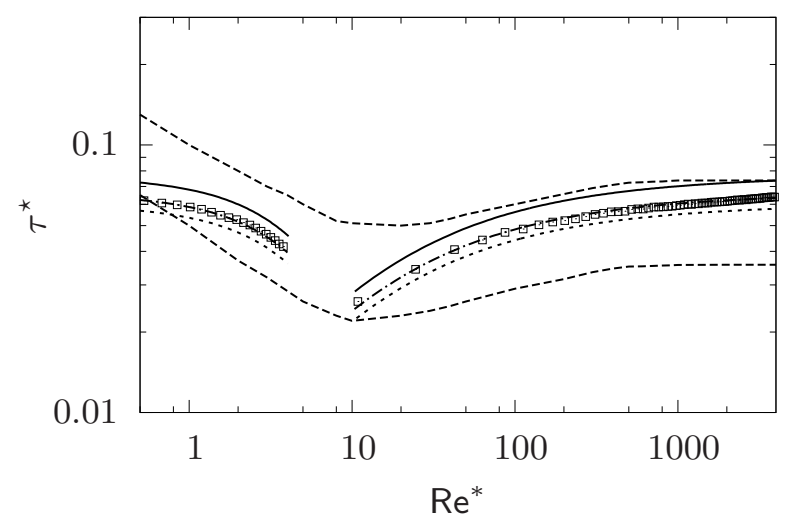

(c)

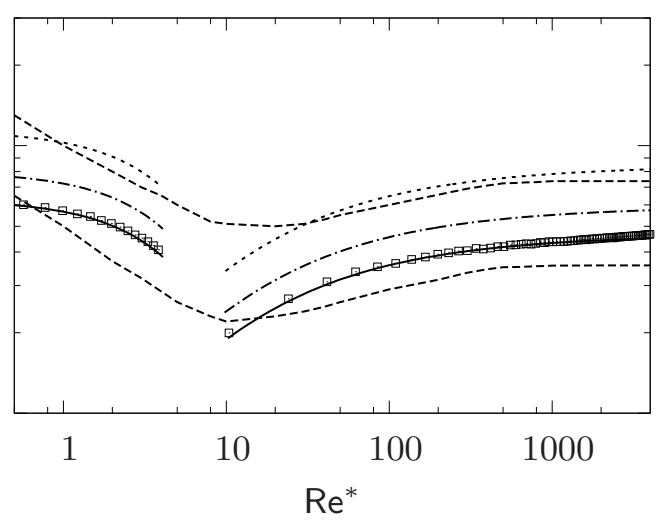

(b)

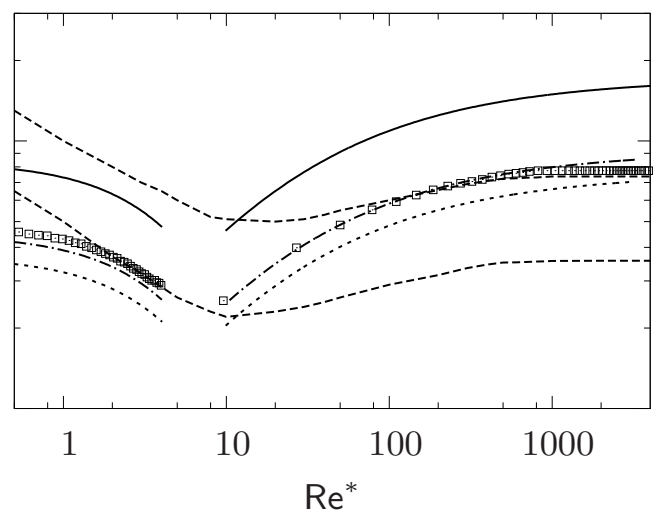

(d)

An. Rolling

An. Slid.

An. Slid."sc"-

Num. DEM

Exp. Shields---------

Figure $14 \mathrm{Re}^{\star}, \tau^{\star}$ relation for $\alpha=15^{\circ}$ and $f=1.0,0.75,0.50,0.25$, (a), (b), (c), (d) respectively.

taking into account the change of mode of movement and shear stresses due to the variation of orientation and position of the particle. Numerical approach has shown that subsequent kind of motion is independent of the flow regime and is usually a combination of modes due to the change of kinematics of the particle.

The discrete approach can be improved by using two way coupling between particle model and flow model. To consider multiple layers of particles with random orientations and shapes, a refined updating of dynamical conditions is necessary once relevant shape changes occur in the domain. 


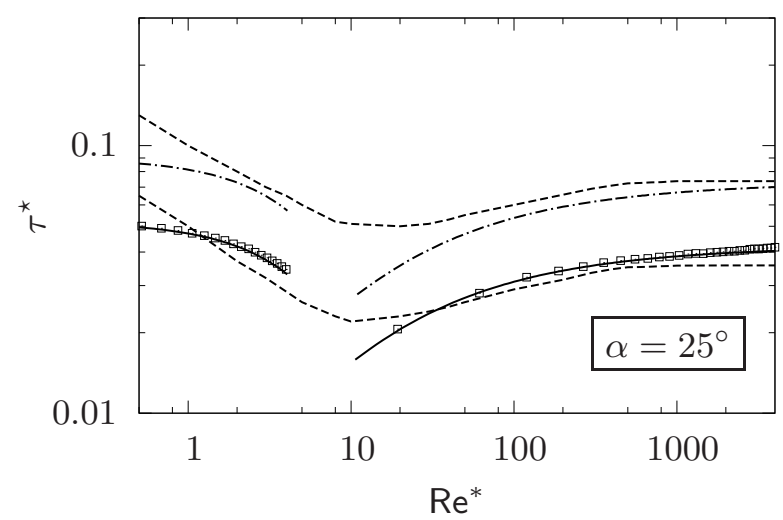

(a)

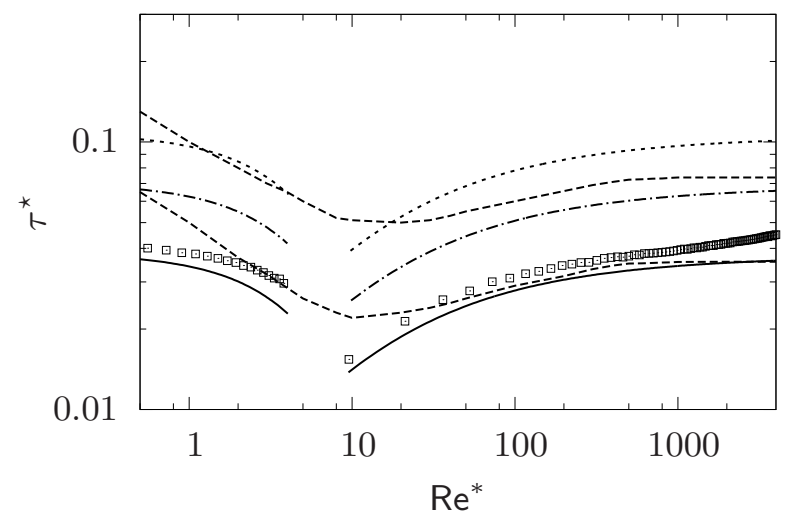

(c)

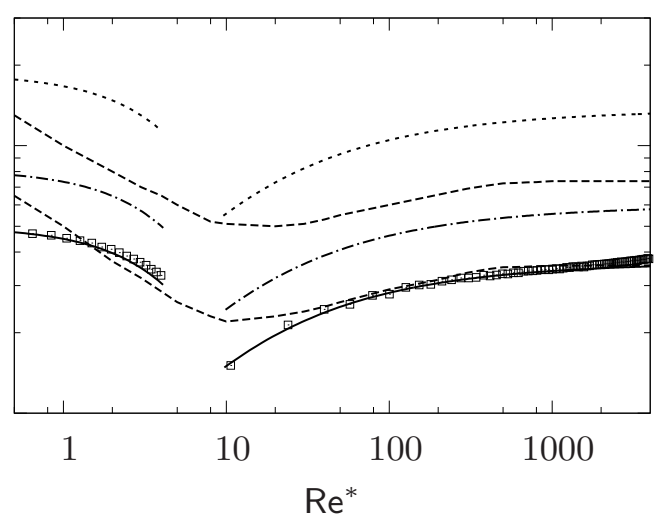

(b)

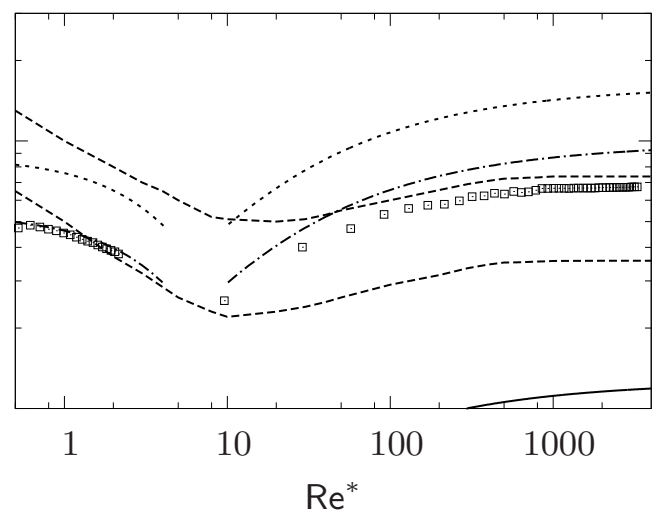

(d)

An. Rolling

An. Slid.

An. Slid."sc"

Num. DEM $\square \square \square \square$

Exp. Shields---------

Figure $15 \mathrm{Re}^{\star}, \tau^{\star}$ relation for $\alpha=25^{\circ}$ and $f=1.0,0.75,0.50,0.25$, (a), (b), (c), (d) respectively. 


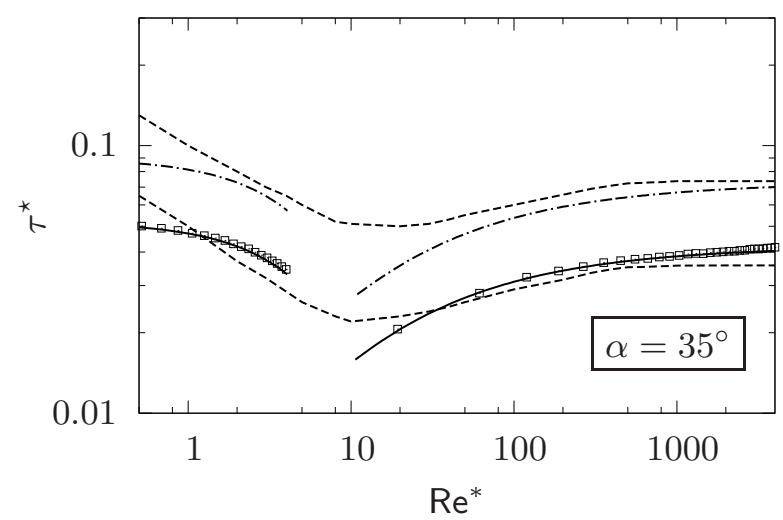

(a)

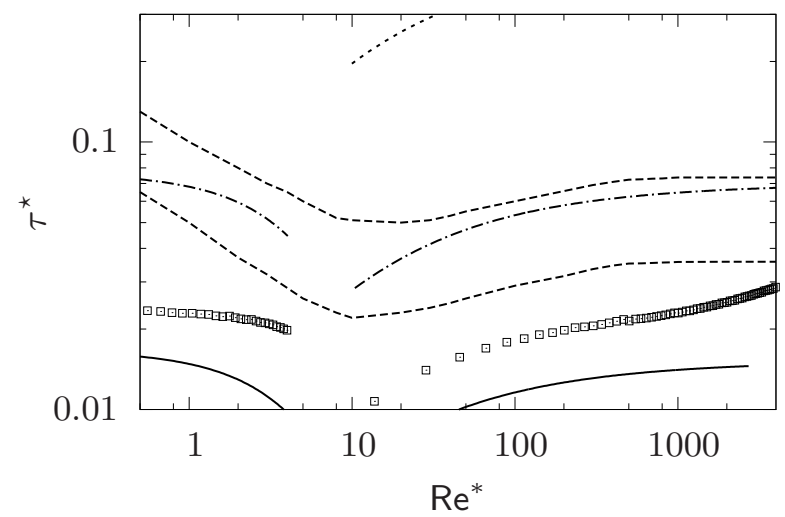

(c)

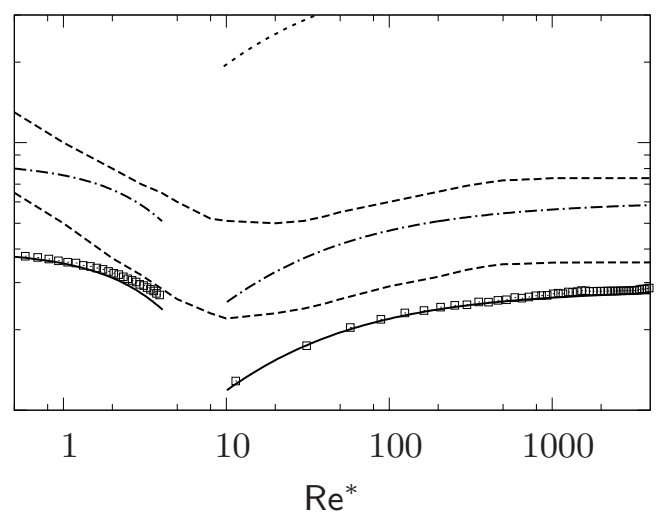

(b)

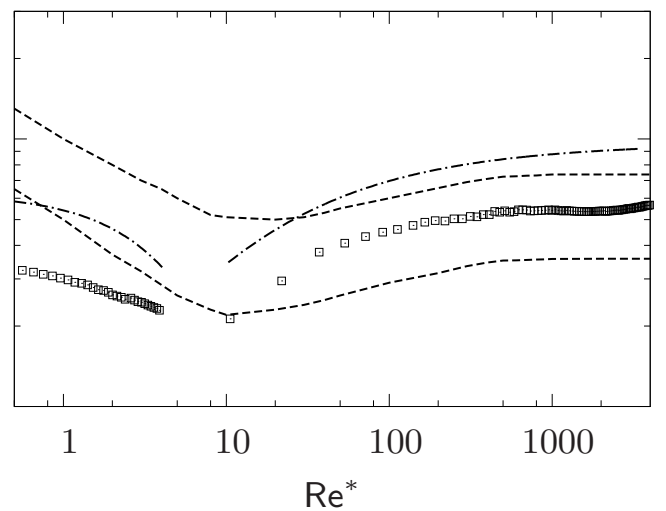

(d)

An. Rolling —— An. Slid. …..... An. Slid."sc"-..-... Num. DEM $\quad$ A

Figure $16 \mathrm{Re}^{\star}, \tau^{\star}$ relation for $\alpha=35^{\circ}$ and $f=1.0,0.75,0.50,0.25$, (a), (b), (c), (d) respectively. 


\section{Fundings}

This work was supported by the MICINN Grant \#BIA-2012-32918 and by the MICINN Grant \#BIA-2015-64994-P (MINECO/FEDER).

\section{Notation}

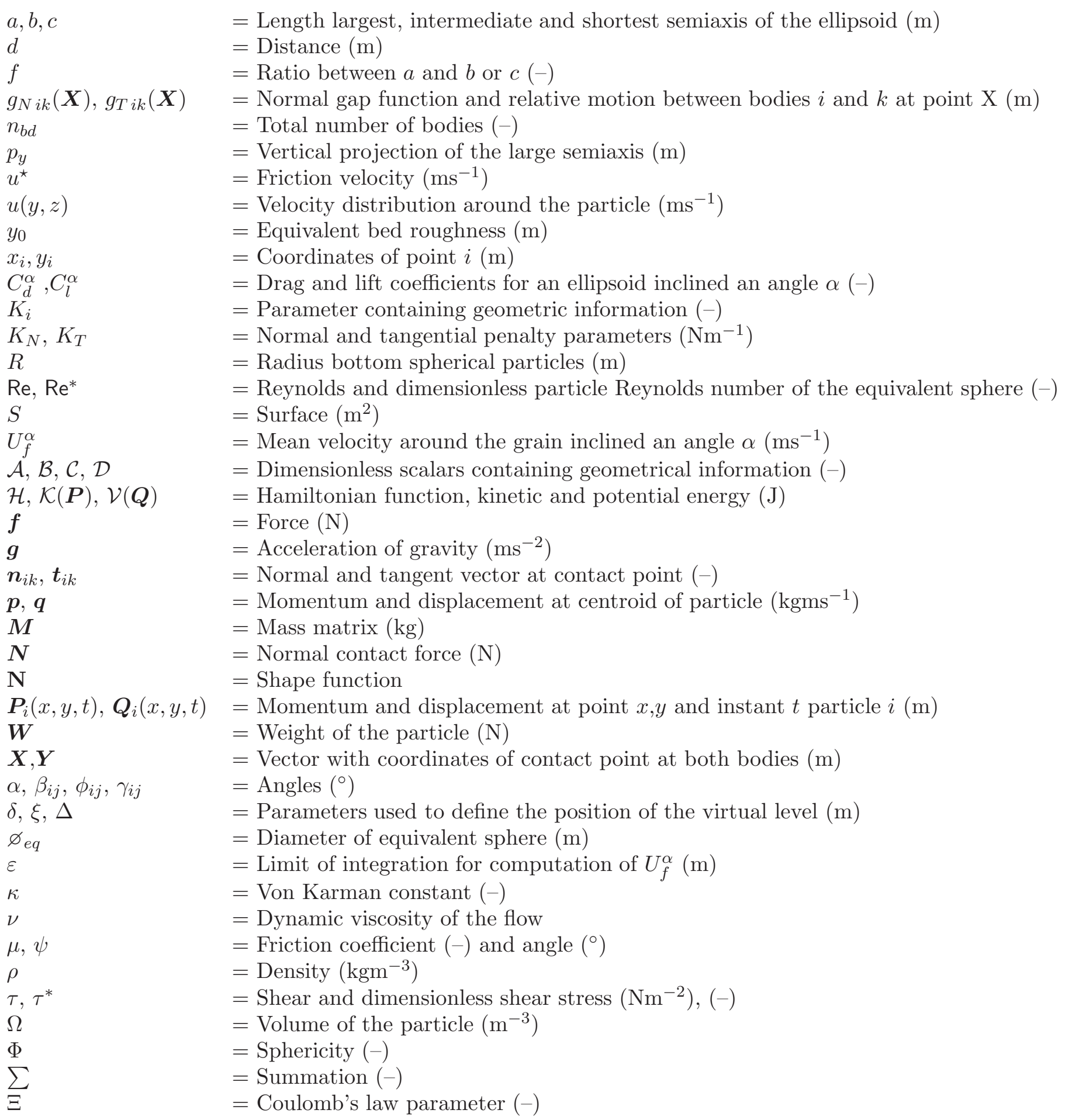




\section{Appendix A. Geometry of the ellipsoid resting over two cylinders}

The unknown angles $\beta_{12}, \beta_{13}, \gamma_{12}, \gamma_{13}$, and distances $d_{12}$ and $d_{13}$, from Eqs. (1), (4), and (6) are computed by imposing that distances $\overline{A B}, \overline{B C}, \overline{C D}, \overline{D E}$, and $\overline{E A}$ form a polygon (Fig. 2),

$$
\left.\begin{array}{l}
R \cos \left(\gamma_{13}-\alpha\right)+d_{13} \cos \left(\beta_{13}-\alpha\right)+d_{12} \cos \left(\beta_{12}+\alpha\right)+R \cos \left(\gamma_{12}+\alpha\right)=2 R \\
R \sin \left(\gamma_{13}-\alpha\right)+d_{13} \sin \left(\beta_{13}-\alpha\right)-d_{12} \sin \left(\beta_{12}+\alpha\right)-R \sin \left(\gamma_{12}+\alpha\right)=0
\end{array}\right\}
$$

The system of equations is complemented with the following relations (Fig. A1),

$$
\begin{gathered}
\beta_{i j}=\arctan \left(f \tan \phi_{i j}\right) ; \quad \gamma_{i j}=\arctan \left(\frac{1}{f} \tan \phi_{i j}\right) ; \quad \beta_{i j}=\arctan \left(f^{2} \tan \gamma_{i j}\right) ; \\
d_{i j}=a \sqrt{\cos ^{2} \phi_{i j}+f^{2} \sin ^{2} \phi_{i j}}=\frac{a \sec \beta_{i j}}{\sqrt{1+\frac{\tan ^{2} \beta_{i j}}{f^{2}}}}=a \sqrt{\frac{1+f^{4} \tan ^{2} \gamma_{i j}}{1+f^{2} \tan ^{2} \gamma_{i j}}}
\end{gathered}
$$

where $\phi_{i j}$ is an auxiliary angle not used in the balances of forces. By combining Eqs. (A1) and (A2), coordinates of the centre of $i$ particle can be written as

$$
x_{i}=R \cos \left(\gamma_{13}-\alpha\right)+d_{13} \cos \left(\beta_{13}-\alpha\right) ; y_{i}=R \sin \left(\gamma_{13}-\alpha\right)+d_{13} \sin \left(\beta_{13}-\alpha\right) .
$$

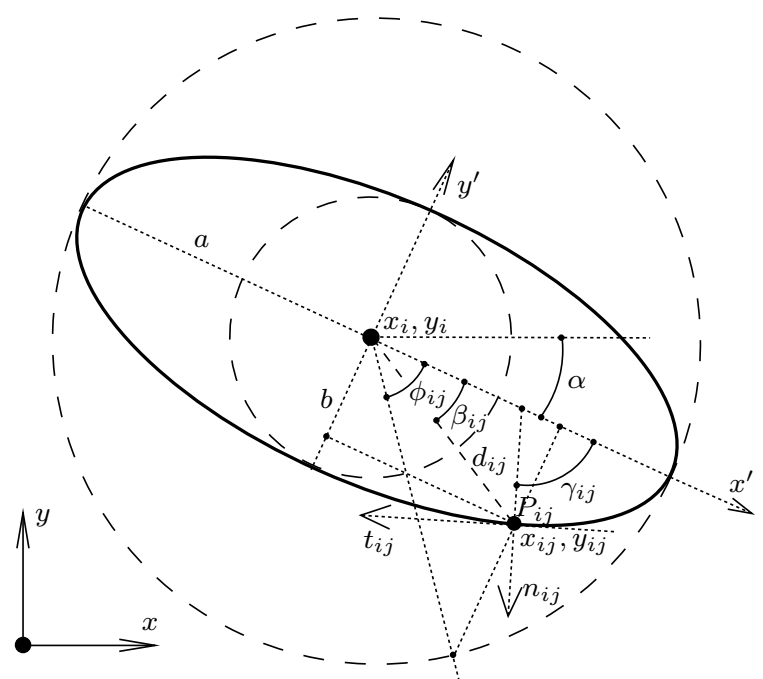

Figure A1 Geometry of an ellipse defined by semiaxis $a, b$, inclination $\alpha$.

The value of $p_{y}$, distance between two horizontal tangents (see Fig. 4) is calculated by imposing $\gamma_{1 t}=\pi / 2-\alpha$ and $\gamma_{1 b}=-\pi / 2-\alpha$, and by using the second of Eqs. (A2), $\phi_{1 t}=\arctan (f / \tan \alpha)$, and $\phi_{1 b}=\arctan (-f / \tan \alpha)$. Finally, $p_{y}=y_{1 t}-y_{1 b}$, where $y_{1 t}=a\left(\cos \phi_{1 t} \sin \alpha+f \sin \phi_{1 t} \cos \alpha\right)$, and $y_{1 b}=a\left(\cos \phi_{1 b} \sin \alpha+f \sin \phi_{1} b \cos \alpha\right)$, respectively. 


\section{References}

Belytschko, T., Liu, W., \& Moran, B. (2000). Nonlinear finite elements for continua and structures. Wiley.

Belytschko, T., \& Neal, M. O. (1991). Contact-impact by the pinball algorithm with penalty and lagrangian methods. International Journal for Numerical Methods in Engineering, 31(3), $547-572$.

Bravo, R., Ortiz, P., \& Pérez-Aparicio, J. (2014). Incipient sediment transport for non-cohesive landforms by the discrete element method (dem). Applied Mathematical Modelling, 38(4), $1326-1337$.

Bravo, R., Pérez-Aparicio, J., \& Gómez-Hernández, J. (2015). Numerical sedimentation particlesize analysis using the discrete element method. Advances in Water Resources, 86, Part A, $58-72$.

Bravo, R., Pérez-Aparicio, J., \& Laursen, T. (2012). An energy consistent frictional dissipating algorithm for particle contact problems. International Journal for Numerical Methods in Engineering, 92(9), 753-781.

Buffington, J. M., \& Montgomery, D. R. (1997). A systematic analysis of eight decades of incipient motion studies, with special reference to gravel-bedded rivers. Water Resources Research, 33(8), 1993-2029. Retrieved from http://dx.doi.org/10.1029/96WR03190 doi:

Cheng, N. S., \& Chiew, Y.-M. (1999). Incipient sediment motion with upward seepage. Journal of Hydraulic Research, 37(5), 665-681.

Chiew, Y.-M., \& Parker, G. (1994). Incipient sediment motion on non-horizontal slopes. Journal of Hydraulic Research, 32(5), 649-660.

Derksen, J. J. (2015). Simulations of granular bed erosion due to a mildly turbulent shear flow. Journal of Hydraulic Research, 53(5), 622-632.

Dey, S. (1999). Sediment threshold. Applied Mathematical Modelling, 23(5), 399-417.

Dey, S. (2003). Threshold of sediment motion on combined transverse and longitudinal sloping beds. Journal of Hydraulic Research, 41(4), 405-415.

Dey, S., Sarker, H. K. D., \& Debnath, K. (1999). Sediment threshold under stream flow on horizontal and sloping beds. Journal of Engineering Mechanics-ASCE, 125(5), 545-553.

Holzer, A., \& Sommerfeld, M. (2008). New simple correlation formula for the drag coefficient of non-spherical particles. Powder Technology, 184(3), 361-365.

James, C. S. (1990). Prediction of entrainment conditions for nonuniform, noncohesive sediments. Journal of Hydraulic Research, 28(1), 25-41.

Ji, C., Munjiza, A., Avital, E., Ma, J., \& Williams, J. J. R. (2013). Direct numerical simulation of sediment entrainment in turbulent channel flow. Physics of Fluids, 25(5), 056601.

Klamkin, M. (1971). Elementary approximations to the area of n-dimensional ellipsoids. American Mathematical Monthly, 78(3), 280-283.

Laursen, T. A. (2002). Computational contact and impact mechanics. Springer-Verlag.

Mando, M., \& Rosendahl, L. (2010). On the motion of non-spherical particles at high reynolds number. Powder Technology, 202(1-3), 1-13.

Mantz, P. A. (1977). Incipient transport of fine grains and flakes by fluids - extended shields diagram. Journal of Hydraulic Division ASCE, 103(6), 601-615.

Miller, M. C., Mccave, I. N., \& Komar, P. D. (1977). Threshold of sediment motion under unidirectional currents. Sedimentology, 24(4), 507-527.

Mohtar, W. H. M. W., \& Munro, R. J. (2013). Threshold criteria for incipient sediment motion on an inclined bedform in the presence of oscillating-grid turbulence. Physics of Fluids, 25(1), 015103.

Ortiz, P., \& Smolarkiewicz, P. K. (2006). Numerical simulation of sand dune evolution in severe winds. International Journal for Numerical Methods in Fluids, 50(10), 1229-1246.

Ortiz, P., \& Smolarkiewicz, P. K. (2009). Coupling the dynamics of boundary layers and evolu- 
tionary dunes. Phys. Rev. E, 79(4), 041307-1.

Rijn, L. V. (1984). Sediment transport. part i: bed load transport. Journal of Hydraulic Engineering-ASCE, 110(10), 1431-1456.

Shi, G., \& Goodman, R. (1985). Two dimensional discontinuous deformation analysis. International Journal for Numerical and Analytical Methods in Geomechanics, 9(6), 541-556.

Shields, A. (1936). Application of similarity principles and turbulence research to bed-load movement (Tech. Rep.). Lab. for Hydraulic Water Resources.

Vowinckel, B., Jain, R., Kempe, T., \& Fröhlich, J. (2016). Entrainment of single particles in a turbulent open-channel flow: a numerical study. Journal of Hydraulic Research, 1-14.

Wellmann, C., Lillie, C., \& Wriggers, P. (2008). A contact detection algorithm for superellipsoids based on a contact detection algorithm for superellipsoids based on the common-normal concept. Engineering Computations, 25(5), 432-442.

Wiberg, P. L., \& Smith, J. D. (1985). A theoretical model for saltating grains in water. Journal of Geophysical Research, 90(C4), 7341-7354.

Yalin, M. S., \& Karahan, E. (1979). Inception of sediment transport. Journal of Hydraulic Division $A S C E, 105(11), 1433-1443$. 